\title{
Influence of air flow parameters on nanosecond repetitively pulsed discharges in a pin-annular electrode configuration
}

\author{
Sylvain A. Heitz ${ }^{1}$ \\ ${ }^{1}$ Laboratoire EM2C, CNRS, CentraleSupélec, Université Paris-Saclay, Grande Voie \\ des Vignes, 92295 Châtenay-Malabry Cedex, France \\ E-mail: sylvain.heitz@ecp.fr \\ Jonas P. Moeck ${ }^{2}$ \\ ${ }^{2}$ Institut für Strömungsmechanik und Technische Akustik, Technische Universität \\ Berlin, 10623 Berlin, Germany \\ E-mail: jonas.moeck@tu-berlin.de
}

Thierry Schuller ${ }^{1}$

E-mail: thierry.schuller@ecp.fr

\section{Denis Veynante ${ }^{1}$}

E-mail: denis.veynante@ecp.fr

\section{Deanna A. Lacoste ${ }^{3}$}

${ }^{3}$ Clean Combustion Research Center, King Abdullah University of Science and Technology, Thuwal 23955-6900, Saudi Arabia

E-mail: deanna.lacoste@kaust.edu.sa

\begin{abstract}
.
The effect of various air flow parameters on the plasma regimes of nanosecond repetitively pulsed (NRP) discharges is investigated at atmospheric pressure. The two electrodes are in a pin-annular configuration, transverse to the mean flow. The voltage pulses have amplitudes up to $15 \mathrm{kV}$, a duration of $10 \mathrm{~ns}$ and a repetition frequency ranging from 15 to $30 \mathrm{kHz}$. The NRP corona to NRP spark (C-S) regime transition and the NRP spark to NRP corona (S-C) regime transition are investigated for different steady and harmonically oscillating flows. First, the strong effect of a transverse flow on the C-S and S-C transitions, as reported in previous studies, is verified. Second, it is shown that the azimuthal flow imparted by a swirler does not affect the regime transition voltages. Finally, the influence of low frequency harmonic oscillation of the air flow, generated by a loudspeaker, is studied. A strong effect of frequency and amplitude of the incoming flow modulation on the NRP plasma regime is observed. Results are interpreted based on the cumulative effect of the NRP discharges and an analysis of the residence times of fluid particles in the inter-electrode region.
\end{abstract}


Keywords: non-equilibrium plasma, acoustic forcing, discharge regime transition, atmospheric pressure.

\section{Nomenclature}

\begin{tabular}{|c|c|c|}
\hline Name & Description & Unit \\
\hline NRP & Nanosecond Repetitively Pulsed & \\
\hline$P R F$ & Pulse Repetition Frequency & $\mathrm{kHz}$ \\
\hline RMS & Root Mean Square & . \\
\hline $\mathrm{C}-\mathrm{S}$ & NRP corona to spark transition & . \\
\hline $\mathrm{S}-\mathrm{C}$ & NRP spark to corona transition & . \\
\hline$C$ & Capacity of the circuit & $\mathrm{F}$ \\
\hline$C_{a}$ & Speed of sound & $\mathrm{m} \cdot \mathrm{s}^{-1}$ \\
\hline$d$ & Gap distance between electrodes & $\mathrm{mm}$ \\
\hline$D_{h}$ & Hydraulic diameter of inter-electrode area & $\mathrm{mm}$ \\
\hline$E$ & Electric field & V.cm ${ }^{-1}$ \\
\hline$E_{p}$ & Energy deposited by a plasma pulse & $\mu \mathrm{J}_{\mathrm{pulse}}{ }^{-1}$ \\
\hline$E_{\text {transition }}$ & $\begin{array}{l}\text { Total deposited energy by the high-voltage } \\
\text { pulses to reach a regime transition }\end{array}$ & $\mu \mathrm{J}$ \\
\hline$f_{a c}$ & Acoustic frequency & $\mathrm{Hz}$ \\
\hline$h$ & Height of inter-electrode area & $\mathrm{mm}$ \\
\hline$I$ & Current measured by the probe & $\mathrm{A}$ \\
\hline$n$ & Number of applied pulses on air particles & . \\
\hline$N$ & Gas density & particles.cm ${ }^{-3}$ \\
\hline$p$ & Pressure & $\mathrm{Pa}$ \\
\hline$P^{*}$ & Non-dimensional power of the pulses & . \\
\hline$r$ & Radial position & $\mathrm{mm}$ \\
\hline$R$ & Radius of inter-electrode area & $\mathrm{mm}$ \\
\hline$R_{\text {air }}$ & Air electrical resistance & $\Omega$ \\
\hline$R e$ & Reynolds number in the nozzle & . \\
\hline$S$ & Swirl number & . \\
\hline St & Strouhal number & . \\
\hline$t$ & Time & $\mathrm{s}$ \\
\hline$t_{R}$ & Air residence time in inter-electrode area & $\mathrm{ms}$ \\
\hline$T$ & Forcing period of the signal & $\mathrm{s}$ \\
\hline$U$ & Voltage measured by the probe & $\mathrm{kV}$ \\
\hline$u_{z}$ & Instantaneous axial velocity & $\mathrm{m} \cdot \mathrm{s}^{-1}$ \\
\hline$u_{\theta}$ & Instantaneous azimuthal velocity & $\mathrm{m} \cdot \mathrm{s}^{-1}$ \\
\hline $\bar{u}_{z}$ & Mean axial velocity & $\mathrm{m} \cdot \mathrm{s}^{-1}$ \\
\hline $\bar{u}_{\theta}$ & Mean azimuthal velocity & $\mathrm{m} \cdot \mathrm{s}^{-1}$ \\
\hline$u_{z}^{\prime}$ & RMS axial velocity & $\mathrm{m} \cdot \mathrm{s}^{-1}$ \\
\hline$u_{z}^{\prime} / \bar{u}_{z}$ & Relative RMS axial velocity & \\
\hline
\end{tabular}




$\begin{array}{lll}\nu_{a i r} & \text { Kinematic viscosity of air } & \mathrm{m}^{2} \cdot \mathrm{s}^{-1} \\ z & \text { Axial position of air particle } & \mathrm{mm} \\ Z_{0} & \text { Characteristic impedance of air } & \mathrm{kg} \cdot \mathrm{m}^{-2} \cdot \mathrm{s}^{-1}\end{array}$

\section{Introduction}

Numerous applications make use of non-equilibrium plasma discharges in an atmospheric pressure air (or air-containing) flow. Among them, a significant number is related to bio-medical applications [1-4], surface processing [5,6], gaseous treatment [7-9] or combustion enhancement [10-12]. Although there is a strong mutual coupling between gaseous flow and plasma, most academic and industrial studies mainly focus on the effect of plasma on the flow.

One of the most spectacular effects of non-equilibrium plasmas on gas dynamics is the generation of ionic wind. Numerous different configurations were studied in order to increase the velocity of the ionic wind, also called electric wind [13-15]. The physical mechanism of this effect has also been modeled and the electrohydrodynamic (EHD) force can be predicted $[16,17]$. Ionic wind generated by plasma discharges can be used to modify an oscillating air flow. An example has been given by controlling shear layer flow instabilities triggered by acoustic forcing in a turbulent air jet with three types of plasma actuators [18]. This control was achieved through a variation of the phase shift between the signals driving the loudspeaker and the plasma actuator. A change in the velocity profile in the shear layer of the jet was also observed. Spark discharges can also affect the flow by thermal expansion [19,20]. The ultrafast heating of the gas (up to several thousand Kelvin) generates shock waves followed by expansion waves that eventually increase local turbulence.

Although effects of plasma on the flow are known relatively well, effects of the flow on plasma discharges have scarcely been investigated. In argon, several studies [21-23] report on the significant influence of the gas flow on the discharge properties, such as breakdown voltage and gas heating. In low-pressure hydrogen, Chabert et al. [24] found a strong effect of the flow on the dissociation degree of a microwave discharge, but a relatively weak influence on the gas temperature.

In air, few studies $[25,26]$ have shown the significant effect of a transverse air stream on the characteristics of positive corona and dielectric barrier discharges. For example, it was shown that for cold plasmas, the breakdown voltage increases with the bulk velocity and that the momentum transfer can be affected by the direction of the flow. It was also shown that the discharge regime can be affected by the gas velocity. In a study dedicated to the characterization of nanosecond-pulse gliding discharges in air, Zhang et al. [31] showed that by changing the air flow from quiescent to $10 \mathrm{~L} \cdot \mathrm{min}^{-1}$ (corresponding to a bulk velocity of about $4.3 \mathrm{~m} \cdot \mathrm{s}^{-1}$ ), both the breakdown voltage and the discharge mode were affected. Recently, Balek et al. [28] studied the effect of an acoustic field on a negative corona discharge in air at atmospheric pressure. They found 
that an intense acoustic field (with velocity fluctuations up to $25 \mathrm{~m} \cdot \mathrm{s}^{-1}$, in a frequency range from 24 to $100 \mathrm{~Hz}$ ) can significantly affect both the stability and the spatial organization of the negative corona or transient spark discharges. An explanation of their experimental results based on transport properties and thermal instabilities of the plasma was proposed. However, all these previous studies on the effect of an air flow on the discharge behavior dealt with relatively low power plasmas. Typically, with a current up to $30 \mathrm{~mA}$ and a minimal thermal impact, the corona or dielectric barrier discharges investigated have limited relevance for applications such as plasma-assisted combustion. To the best of our knowledge, the impact of transverse flows and acoustic waves on the organization and regime transition of non-equilibrium thermal air plasmas such as Nanosecond Repetitively Pulsed (NRP) spark discharges, laser sparks or radiofrequency discharges has never been investigated.

In this paper, we focus on the influence of a transverse stream, eventually modulated by harmonic acoustic waves, on the breakdown voltage and the regime transitions of NRP discharges in air at atmospheric pressure. Previous studies have shed light upon key parameters governing the regime of NRP plasma discharges. The gas temperature, the inter-electrode gap distance, the Pulse Repetition Frequency $(P R F)$, as well as the electrode geometry have been established as relevant parameters [29]. In this work, effects of a steady laminar air stream with a Reynolds number lower than 1300 is first assessed by investigating the NRP corona to spark (C-S) and the NRP spark to corona (S-C) transition voltages dependence to changes of the axial or azimuthal velocity components of the incoming flow. The effect of axial acoustic forcing is then examined.

\section{Experimental setup}

\subsection{The test-rig}

The experiments were performed in an atmospheric pressure test-rig shown in Figure 1. A flow of air at room temperature, controlled by a mass flowmeter (Bronkhorst EL-Flow) enters the experimental setup at its bottom. The air flows through a perforated plate and a honeycomb which are not shown in Figure 1. It then enters a converging section. These elements are used to laminarize the flow and reduce the boundary layer thickness leading to a top hat velocity profile at the converging nozzle outlet. An element with or without radial swirling vanes is also placed in between the converging nozzle and the cylindrical tube to set the flow in rotation in the azimuthal direction or not. Three swirlers featuring different blade angles: $0^{\circ}$ (no rotation of the flow), $34^{\circ}$ and $48^{\circ}$ were used in these experiments. The element carries also a small central cylindrical bluff body of $2.6 \mathrm{~mm}$ diameter made of stainless steel, which is flush-mounted with the injection tube outlet. With a blade angle of $0^{\circ}$ a purely axial flow is generated, corresponding to the reference case of this study. The two other blade angles were chosen in order to generate swirl levels with swirl numbers $S$ (defined as the ratio of the axial flux of 


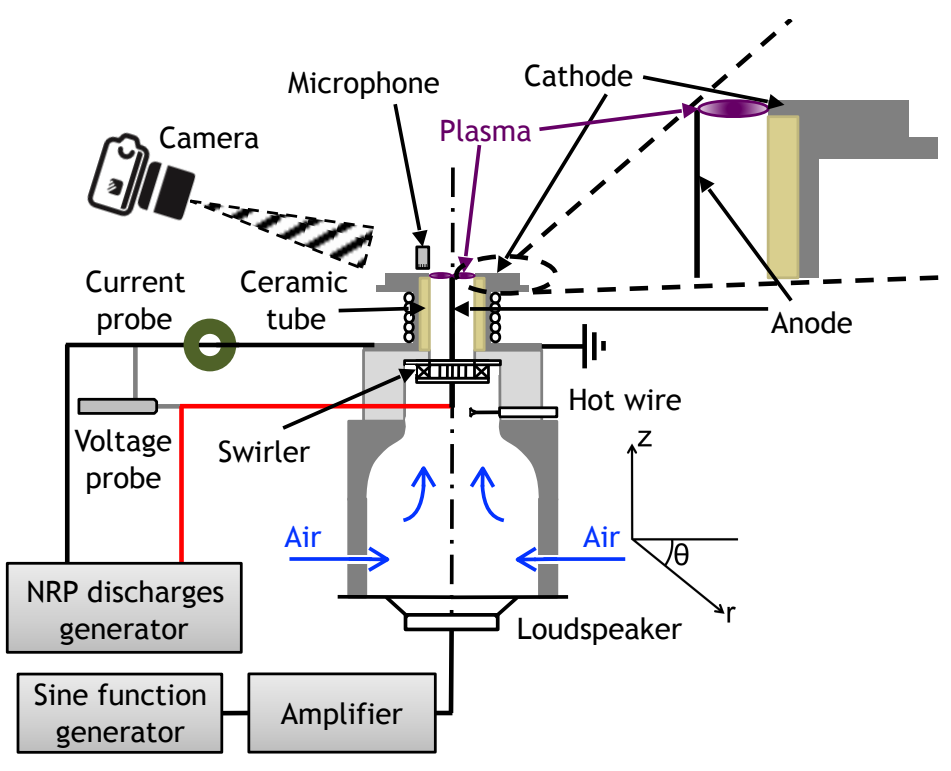

Figure 1. Schematic of the experimental setup and zoom on the inter-electrode area.

angular momentum to the axial flux of axial momentum [30]) of 0.5 and 0.7 . These values are known to ease stabilization of swirling premixed flames and will allow future studies of plasma-assisted combustion.

The NRP plasma discharges are generated between two stainless steel electrodes, in a pin-annular configuration. Figure 1 provides a zoom of the inter-electrode area to allow a better description of the electrode configuration. The anode corresponds to the central bluff body described above. The cathode is an annular ring of $D=$ $17.9 \mathrm{~mm}$ diameter and $h=1.2 \mathrm{~mm}$ height. The gap distance between the anode and the cathode is therefore $7.7 \mathrm{~mm}$. Upstream of the cathode, a cylindrical piece of ceramic with the same external diameter $(D=17.9 \mathrm{~mm})$ is flush-mounted with the metallic ring to prevent parasitic discharges inside the injection tube. Both electrodes are connected to a FPG Series high-voltage pulse generator (FID Technologies). The pulses have an amplitude in the range of 3.5 to $15 \mathrm{kV}$, last $10 \mathrm{~ns}$ with a pulse repetition frequency that has been set to either $P R F=15$ or $30 \mathrm{kHz}$.

A loudspeaker (Monacor SPH-115, $80 \mathrm{~W}$ power) at the bottom of the setup is connected to a signal synthesizer (TGA1200 Series, Aim \& TTi) and a Hi-Fi amplifier (RA-04, Rotel). These elements are used to generate harmonic acoustic waves at controlled amplitudes and frequencies. The forcing frequency is varied between $f_{a c}$ $=16$ and $192 \mathrm{~Hz}$, and the velocity amplitude in the inter-electrode region is evaluated by using a hot wire and ranges from $u_{z}^{\prime}=0$ to $3 \mathrm{~m} \cdot \mathrm{s}^{-1}$.

\subsection{Instrumentation}

Figure 2 presents a typical pulse measurement obtained for NRP corona discharges. The temporal evolution of a voltage pulse, the corresponding total current, and associated energy are shown. Electrical measurements were performed with a voltage probe (PPE 
$20 \mathrm{kV}$, LeCroy) and a current probe (Model 6585, Pearson Electronics) attenuated with a low-voltage attenuator (2-20, Barth Electronics). The electrical signals are recorded with an oscilloscope (1 GHz WavePro 7000A Series, LeCroy). Voltage and current signals were first synchronized. The voltage and conduction current waveforms (obtained after subtracting the capacitive current from the total current) were then multiplied and integrated to obtain the energy deposition per pulse $[35,36]$.

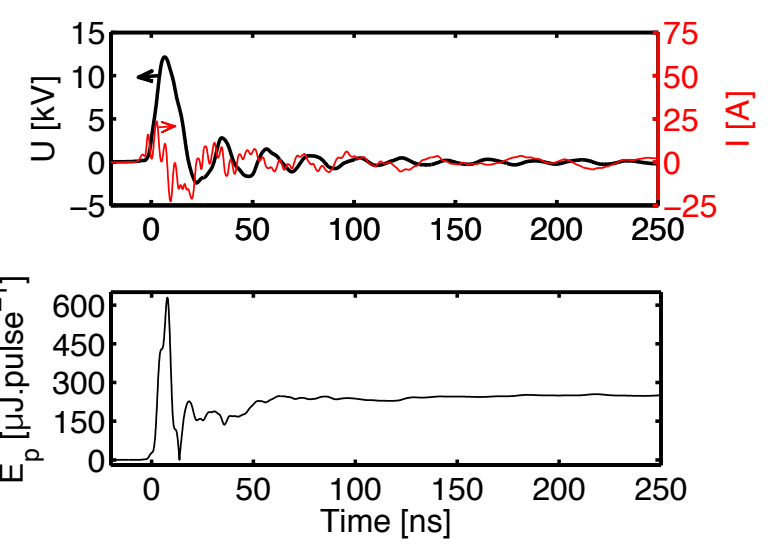

Figure 2. Example of voltage and current waveforms and corresponding deposited energy obtained during a pulse of a NRP corona discharge. The $P R F$ is set to $30 \mathrm{kHz}$, and the air bulk flow velocity, $\bar{u}_{z}$, is $7.65 \mathrm{~m} \cdot \mathrm{s}^{-1}$.

Imaging of the discharge organization in the inter-electrode region is realized by collecting the plasma light emission in the visible range with a camera (D7000, Nikon) equipped with a glass objective. The exposure time is between $0.02 \mathrm{~s}$ and $3.20 \mathrm{~s}$, the sensitivity is kept constant and set to ISO 1600 and the aperture is set to f/25.

The velocity of the air flow was measured with a hot wire (55P16, Dantec Dynamics) located upstream of the swirler. The measurement of the velocity in the interelectrode area was obviously not possible with a hot wire during plasma generation. Section 2.3.2 will describe how the velocity in the inter-electrode area is determined from measurements upstream of the swirler.

Finally, acoustic pressure measurements close to the inter-electrode area were made with a microphone (Type 4138, Brüel \& Kjær) and a preamplifier (Type 2670, Brüel \& Kjær) connected to a conditioning amplifier (Type 2690 Nexus, Brüel \& Kjær). Setting the microphone in the manner depicted in Figure 1 is intrusive but will not significantly affect the pressure oscillation in the inter-electrode area.

\subsection{Experimental procedure}

\subsubsection{Steady air flow}

For steady injection conditions, experiments are carried out to analyze the impact of the velocity on the plasma regime. The velocity profile in the inter-electrode area is thus determined when the hot wire is set $2 \mathrm{~mm}$ above the outlet of the injection 
tube. This intrusive sensor is mainly sensitive to the norm of the velocity component of the flow perpendicular to the hot wire. Admitting that the radial velocity component of the swirling flow can be neglected, we oriented the hot wire so as to successively mainly measure the axial, $\bar{u}_{z}$, and azimuthal, $\bar{u}_{\theta}$, velocity components of the swirling jet exhausting the injection tube. These measurements were carried out for the two swirlers with $34^{\circ}$ and $48^{\circ}$ blade angle, designated by medium swirl $(S=0.5)$ and strong swirl $(S=0.7)$ in the following. The resulting axial and azimuthal velocities under a steady air flow were calculated according to:

$$
\begin{aligned}
& \bar{u}_{z}=\frac{2}{R^{2} \Delta t} \int_{0}^{R} \int_{0}^{\Delta t} r u_{z}(t, r) d t d r \\
& \bar{u}_{\theta}=\frac{2}{R^{2} \Delta t} \int_{0}^{R} \int_{0}^{\Delta t} r u_{\theta}(t, r) d t d r .
\end{aligned}
$$

where $R=9 \mathrm{~mm}$ is the radius of the inter-electrode area and $\Delta t=16 \mathrm{~s}$ is the signal acquisition time. The axial velocity $\bar{u}_{z}$ is equal to the bulk velocity.

Spatially and temporally averaged velocity components are calculated as follows:

$$
\begin{aligned}
\bar{u}_{z}^{r} & =\frac{2}{R^{2}} \int_{0}^{R} r u_{z}(t, r) d r, & \bar{u}_{z}^{t} & =\frac{1}{\Delta t} \int_{0}^{\Delta t} u_{z}(t, r) d t, \\
\bar{u}_{\theta}^{r} & =\frac{2}{R^{2}} \int_{0}^{R} r u_{\theta}(t, r) d r, & \bar{u}_{\theta}^{t} & =\frac{1}{\Delta t} \int_{0}^{\Delta t} u_{\theta}(t, r) d t .
\end{aligned}
$$

Results are presented in Figure 3 for the same bulk velocity of $1.42 \mathrm{~m} \cdot \mathrm{s}^{-1}$ in the annular gap of the injection tube. The left figure shows the local axial velocity profiles and the right figure presents the local azimuthal velocity profiles for the two tested swirlers. The sign of the signal was adjusted across the symmetry axis of the system for the azimuthal velocity component. The strongly swirled flow reaches higher axial and azimuthal peak velocities than the moderately swirled flow. The axial velocities both feature an M-shaped profile with about the same minimum velocity of about $0.5 \mathrm{~m} \cdot \mathrm{s}^{-1}$ on the burner axis. This velocity defect in the center flow originates from the wake of the central bluff body and from the negative axial pressure gradient due to the swirling flow. The azimuthal velocity component features a Rankine-like vortex profile except near the burner axis. The hot wire is not suited for velocity measurements in this low azimuthal velocity region and the contribution from the axial flow might be of the same order of magnitude. Away from this region, the radial and azimuthal velocity gradients increase between the medium and strong swirl cases. The main observation is that the two swirlers allow to impart a strong azimuthal component to the flow with a peak velocity roughly equal to half the axial peak velocity of the jet.

Measurements of the C-S and S-C regime transition voltages were first made in a configuration with a purely axial flow (swirler with $0^{\circ}$ blade angle, corresponding to $S=0$ ) by changing the flow rate. The range of explored bulk flow velocities, $\bar{u}_{z}$, 

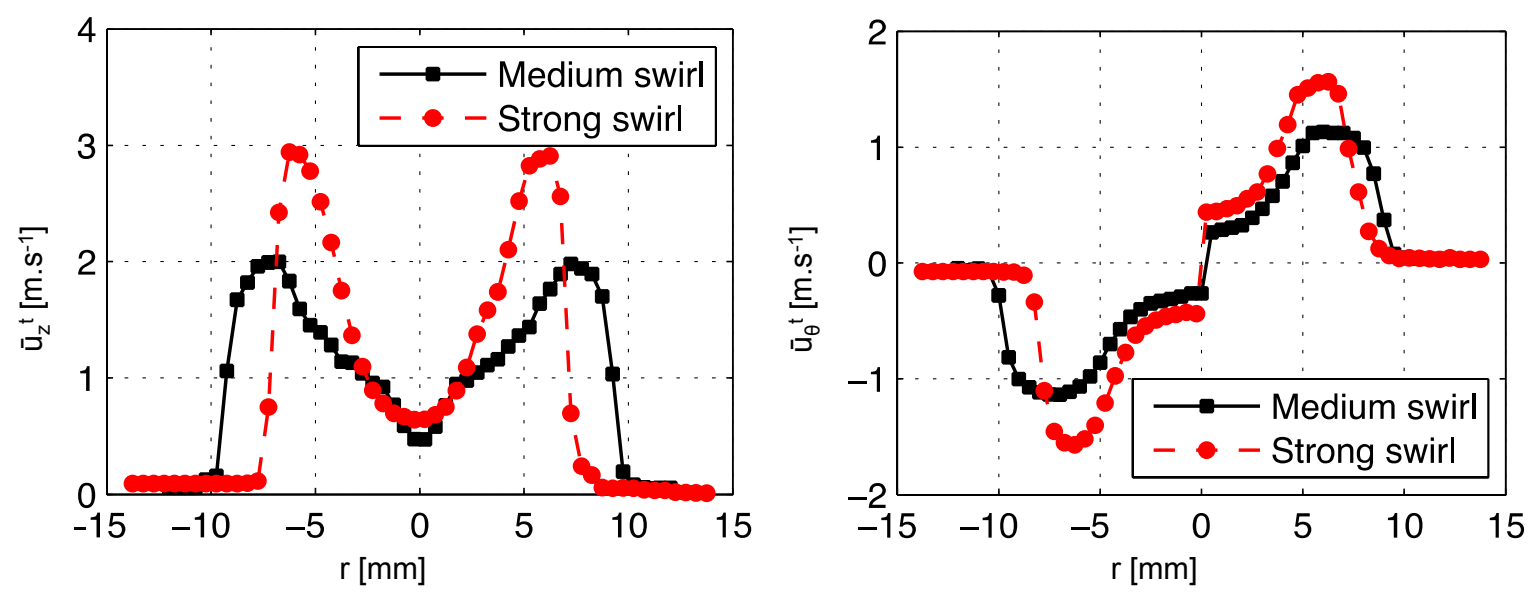

Figure 3. Axial (left) and tangential (right) velocity profiles for two types of swirlers in the test-rig: the medium swirler (solid black lines) and the strong swirler (dashed red lines). The bulk velocity is set to $1.42 \mathrm{~m} \cdot \mathrm{s}^{-1}$.

in the inter-electrode gap is indicated in Table 1 together with the Reynolds number, calculated with:

$$
R e=\frac{\bar{u}_{z} \times D_{h}}{\nu_{a i r}}
$$

where $\bar{u}_{z}$ is the bulk flow velocity, as defined in Equation 1, $D_{h}=15.3 \mathrm{~mm}$ is the hydraulic diameter of the inter-electrode area and $\nu_{\text {air }}=1.59 \times 10^{-5} \mathrm{~m}^{2} \cdot \mathrm{s}^{-1}$ is the kinematic viscosity of air.

Table 1. Velocities in the inter-electrode area for the regime transition voltages study in a steady air flow and corresponding Reynolds numbers.

\begin{tabular}{lr}
\hline $\bar{u}_{z}\left[\mathrm{~m} . \mathrm{s}^{-1}\right]$ & \multicolumn{1}{l}{$\mathrm{Re}$} \\
\hline 0.33 & 318 \\
\hline 0.66 & 636 \\
\hline 1.00 & 963 \\
\hline 1.33 & 1281 \\
\hline
\end{tabular}

For a fixed bulk flow velocity, the inter-electrode voltage is slowly increased, so as to visually reach the $\mathrm{C}-\mathrm{S}$ transition. This transition could not be detected for bulk flow velocities higher than $\bar{u}_{z}=1.33 \mathrm{~m} . \mathrm{s}^{-1}$ due to the limited maximum amplitude of the high voltage pulses of $15 \mathrm{kV}$. The voltage is then decreased until the reverse (S-C) transition takes place. The measurements were repeated thrice and averaged results are presented. The error bars in the following figures correspond to the maximum differences observed between these three measurements. Effect of swirl is then examined in a second set of experiments for two fixed flow rates corresponding to $\bar{u}_{z}=0.33$ and $1.00 \mathrm{~m} \cdot \mathrm{s}^{-1}$. 


\subsubsection{Harmonically oscillating air flow}

Effects of acoustic forcing on the NRP discharge regime are examined without azimuthal velocity of the flow $(S=0)$. The investigated conditions are summarized in Table 2 . The amplitude of the voltage pulses is chosen to obtain NRP corona discharges close to the transition to NRP spark discharges.

Table 2. Sets of experimental conditions used for the determination of the influence of an oscillating flow on NRP plasma discharges.

\begin{tabular}{llll}
\hline $\begin{array}{l}\text { Label of } \\
\text { the set }\end{array}$ & $\begin{array}{l}\bar{u}_{z} \\
{\left[\mathrm{~m}_{\mathrm{s}}{ }^{-1}\right]}\end{array}$ & $\begin{array}{l}P R F \\
{[\mathrm{kHz}]}\end{array}$ & $\begin{array}{l}U \\
{[\mathrm{kV}]}\end{array}$ \\
\hline $1^{\text {st }}$ set & 1.42 & 30 & 12.75 \\
\hline $2^{\text {nd }}$ set & 0.81 & 30 & 12.02 \\
\hline $3^{\text {rd }}$ set & 0.81 & 15 & 13.93 \\
\hline
\end{tabular}

For each set of conditions in Table 2, the amplitude of the acoustic forcing is slowly increased from zero to a level where a C-S transition is observed. If the amplitude is increased further, the plasma regime returns to NRP corona discharges (S-C transition). The hot wire, placed far enough from the walls, at the converging nozzle outlet, is used to deduce the velocity signal in the inter-electrode gap and the corresponding mean and root mean square (RMS) values of this signal for the C-S and S-C transitions. These calculations are based on mass balance for low-frequency acoustic waves leading to a conservation of the acoustic volume flow rate over an axial distance which is short compared to the acoustic wavelength. The cross-sectional area at the converging nozzle outlet being 3.36 times larger than the cross section in the inter-electrode region, the velocity measured at the convergent exhaust needs to be multiplied by 3.36 to determine the velocity in the inter-electrode annulus.

Predictions are compared in Figure 4 with direct measurements in the interelectrode region in the absence of plasma for two forcing frequencies: $f_{a c}=40 \mathrm{~Hz}$ (left) and $f_{a c}=80 \mathrm{~Hz}$ (right). The signals are well superimposed. The values of the deviation between measured signals and reconstructed signals have been systematically analyzed for the whole range of frequencies and forcing amplitudes. The maximum uncertainty induced by the change of the measurement location is $13 \%$.

\section{Results}

\subsection{Steady air flow}

Effects of the air velocity on NRP plasma discharges is first assessed by direct visualizations of the inter-electrode area presented in Figures 5(a) to 5(d), with the camera positioned so as to visualize the inter-electrode area. The inter-electrode voltage is kept constant in these experiments and set to $U=12.46 \mathrm{kV}$; the $P R F$ is set to $30 \mathrm{kHz}$. Figure 5(a) corresponds to a case without flow (within quiescent air). Figures 5(b), (c) 

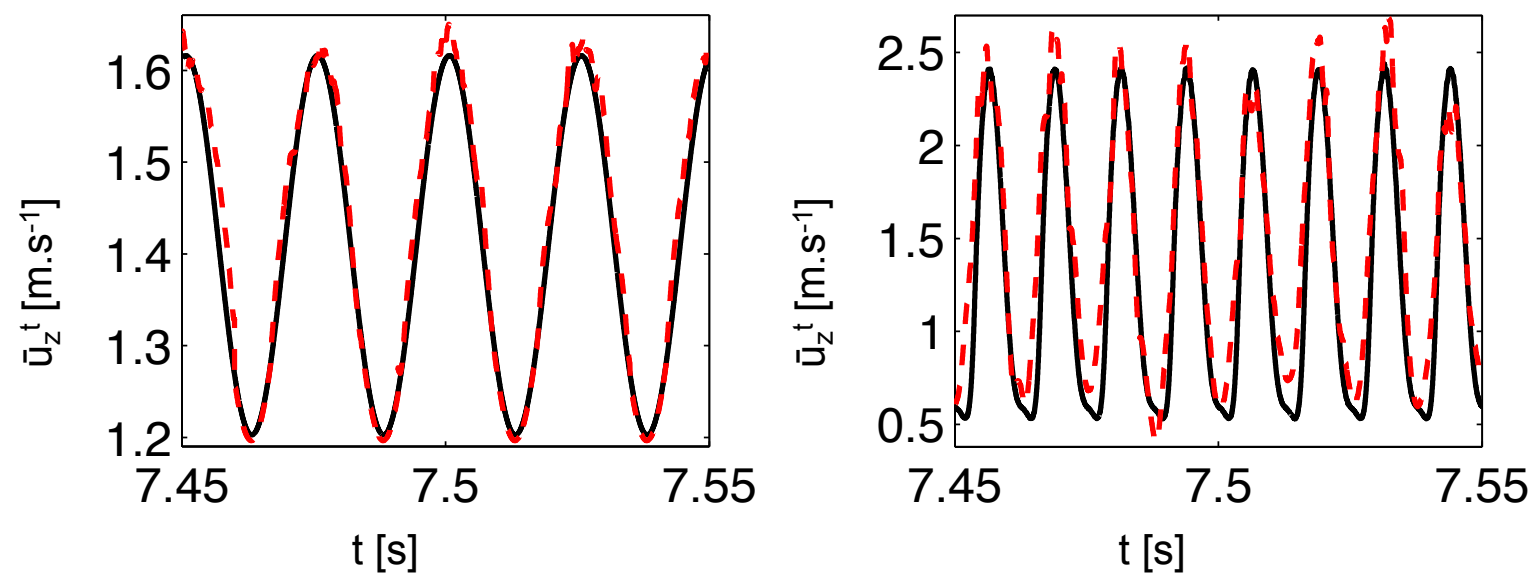

Figure 4. Velocity signals for two acoustic forcing frequencies: $40 \mathrm{~Hz}$ (left) and $80 \mathrm{~Hz}$ (right). The velocity upstream of the swirler (solid black lines) was multiplied by the ratio of cross-sectional areas, 3.36, to compare it with the velocity measured in the inter-electrode area (dashed red lines). The bulk velocity is set to $\bar{u}_{z}=1.42 \mathrm{~m} \cdot \mathrm{s}^{-1}$.

and (d) correspond to observations with a bulk flow velocity $\bar{u}_{z}$ in the inter-electrode area of, respectively, $0.81 \mathrm{~m} . \mathrm{s}^{-1}, 0.81 \mathrm{~m} . \mathrm{s}^{-1}$ and $1.42 \mathrm{~m} . \mathrm{s}^{-1}$. There is no azimuthal velocity in cases (b) and (d) $(S=0)$, and (c) is obtained with medium swirl, $S=0.5$.

In Figure 5(a) (without flow), the NRP plasma discharges are in the spark regime as defined in [34], and are well distributed around the whole surface of the anode. The luminous intensity is higher and the sparks are more uniformly distributed than in Figure 5(b), obtained for a bulk velocity of $\bar{u}_{z}=0.81 \mathrm{~m} \cdot \mathrm{s}^{-1}$. In this case, the discharges mainly start from the edge of the anode and reach the whole surface of the cathode. In Figure 5(c), the NRP spark discharges look similar as in Figure 5(b) but they mainly reach the upper part of the cathode. In these three figures, the exposure time of the camera was set to $0.02 \mathrm{~s}$, allowing 600 pulses at a $P R F$ of $30 \mathrm{kHz}$ to be imaged. In Figure 5(d), the NRP discharges are in the corona regime, as defined in [29], and the exposure time had to be significantly increased (to $3.20 \mathrm{~s}$ ) to obtain a good image quality, corresponding to a visualization of 96000 pulses at the same $P R F$ of $30 \mathrm{kHz}$. In these experiments, it was observed that the NRP discharges transit directly from spark to corona; the intermediate NRP glow regime [29,31] does not appear.

These figures clearly indicate that an air stream and swirl significantly affect the organization as well as the regime of the NRP plasma discharges. An inter-electrode area with no air motion, as in Figure 5(a) favors the presence of NRP spark discharges and their luminous intensity. This can be explained by the fact that a constant air flow, as in Figures 5(b), (c) and (d), prevents the accumulation of the effects of successive plasma pulses, which is one of the key characteristics of NRP discharges (see for example [32,33]). By only considering this phenomenon, it is clear that the residence time of the fluid particles convected by a transverse air flow within the inter-electrode region will determine the number of air particles treated by the plasma pulses (addition 

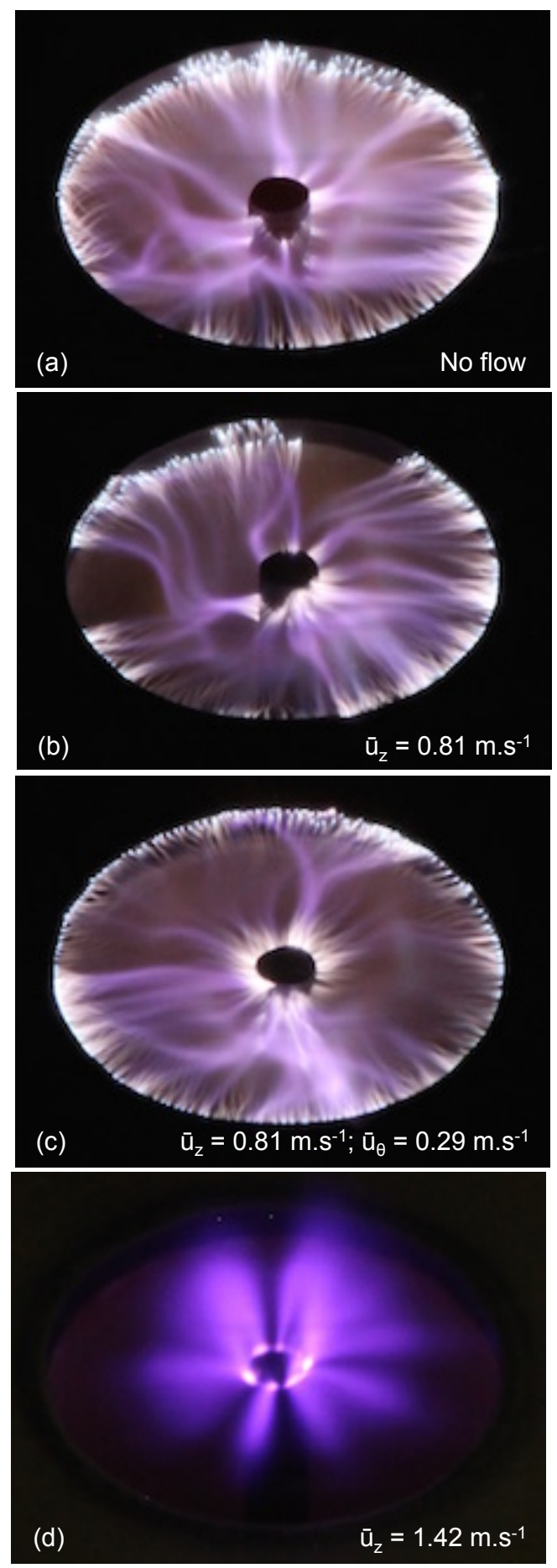

Figure 5. Photographs of the NRP plasma discharges obtained for different flow conditions with pulse voltage amplitude of $12.46 \mathrm{kV}$ and a $P R F$ of $30 \mathrm{kHz}$. Pictures (a), (b) and (c) were taken with an exposure time of $0.02 \mathrm{~s}$, while picture (d) was taken with an exposure time of $3.20 \mathrm{~s}$. (a) $\bar{u}_{z}=0$, (b) $\bar{u}_{z}=0.81 \mathrm{~m} \cdot \mathrm{s}^{-1}, S=0$, (c) $\bar{u}_{z}=0.81$ $\mathrm{m} \cdot \mathrm{s}^{-1}, \mathrm{~S}=0.5$ and $(\mathrm{d}) \bar{u}_{z}=0.81 \mathrm{~m} \cdot \mathrm{s}^{-1}, S=0$.

of heat and active species). If they are transported faster out of the inter-electrode area, this cumulative effect of NRP discharges is reduced, lowering the probability of 
the creation of NRP spark discharges. This scenario is retained here to interpret the following results.

\subsubsection{Impact of an axial stream}

Effects of a mean bulk flow on the C-S and S-C regime transition voltages are presented in Figure 6. The regime transition voltage increases with the bulk air flow velocity. A hysteresis effect is observed, placing the C-S transition curve (solid black line) at a higher voltage level than the S-C transition curve (dashed red line). This hysteresis may be explained by a heating of the anode by the spark discharges. The width of the hysteresis decreases from $3 \mathrm{kV}$ down to $1 \mathrm{kV}$ when the bulk flow velocity increases from 0.33 to $1.33 \mathrm{~m} \cdot \mathrm{s}^{-1}$. This is consistent with a more efficient cooling of the electrode by the surrounding flow, leading to a lower temperature of the gas close to the surface, which is detrimental to the spark regime [29].

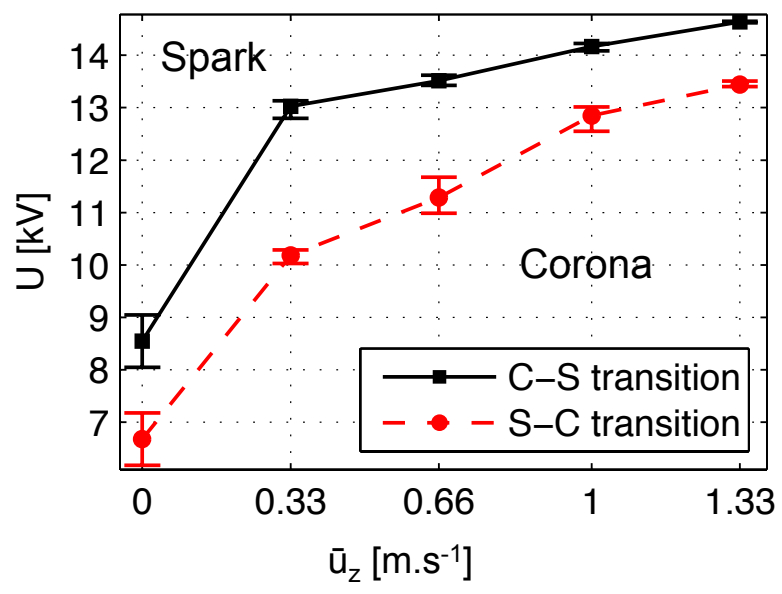

Figure 6. Voltage values for C-S (solid black) and S-C (dashed red) transitions as a function of the axial velocity for a non-swirled air flow $\left(\bar{u}_{\theta}=0\right)$. The $P R F$ is set to $30 \mathrm{kHz}$.

This effect of the bulk flow velocity on the breakdown voltage was already observed by Jaworek and Krupa [25] but for DC corona discharges. The authors suggested that the increase in breakdown voltage according to the air velocity is due to an increase in the effective length of the plasma streamers due to their deflection by the air flow. As in our case the length of the discharge channels does not seem to be affected by the flow, as seen in Figures 5(b) and 5(c), it appears that this explanation does not apply here. These observations are further analyzed in Section 4.1.

To validate the analysis of Section 4.1, the results from Figure 6 are compared with another set of measurements for the C-S transition. This experiment has been performed by setting a constant inter-electrode voltage, $U=14.60 \mathrm{kV}$. With a bulk air velocity set at a value between 0.33 and $1.33 \mathrm{~m} \cdot \mathrm{s}^{-1}$, the $P R F$ is increased until the C-S transition is reached. The results are displayed in Figure 7 and show a linear influence of the axial 
air velocity on the $P R F$ to reach $\mathrm{C}-\mathrm{S}$ transition. Note that, since this experiment has only been performed once, there are no error bar on this figure.

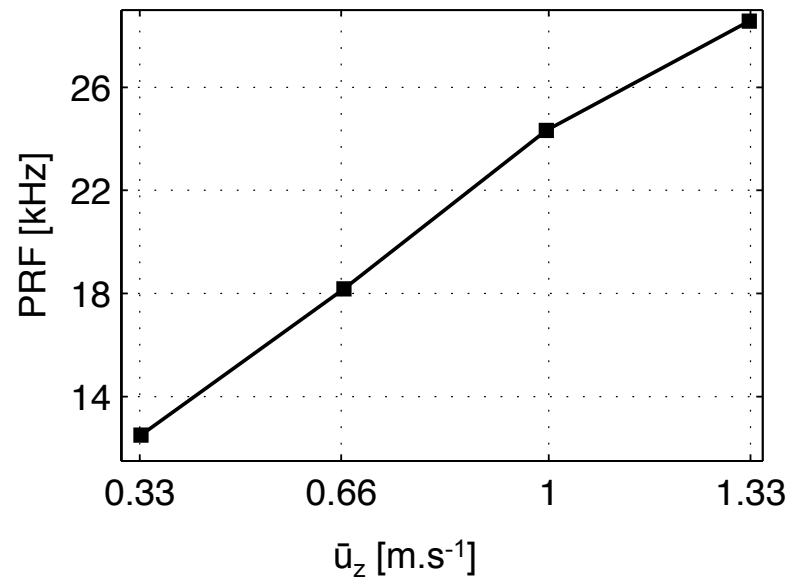

Figure 7. $P R F$ values for $\mathrm{C}-\mathrm{S}$ transition as a function of the bulk flow velocity for a non-swirled flow $\left(\bar{u}_{\theta}=0\right)$. The inter-electrode voltage, $U$, is set to $14.60 \mathrm{kV}$.

\subsubsection{Impact of an azimuthal flow}

Effects of swirl are now analyzed. The C-S and S-C transition voltages are measured in three configurations: without azimuthal velocity, with moderate swirl and with strong swirl. Results are presented for two resulting axial velocities $\bar{u}_{z}=0.33 \mathrm{~m} \cdot \mathrm{s}^{-1}$ and $\bar{u}_{z}=$ $1.00 \mathrm{~m} . \mathrm{s}^{-1}$ in Figure 8. The resulting azimuthal velocity $\bar{u}_{\theta}$ indicated in these figures corresponds to the resulting velocity reached by the azimuthal flow in the swirling jet at the injection tube outlet, obtained with Equation 2.
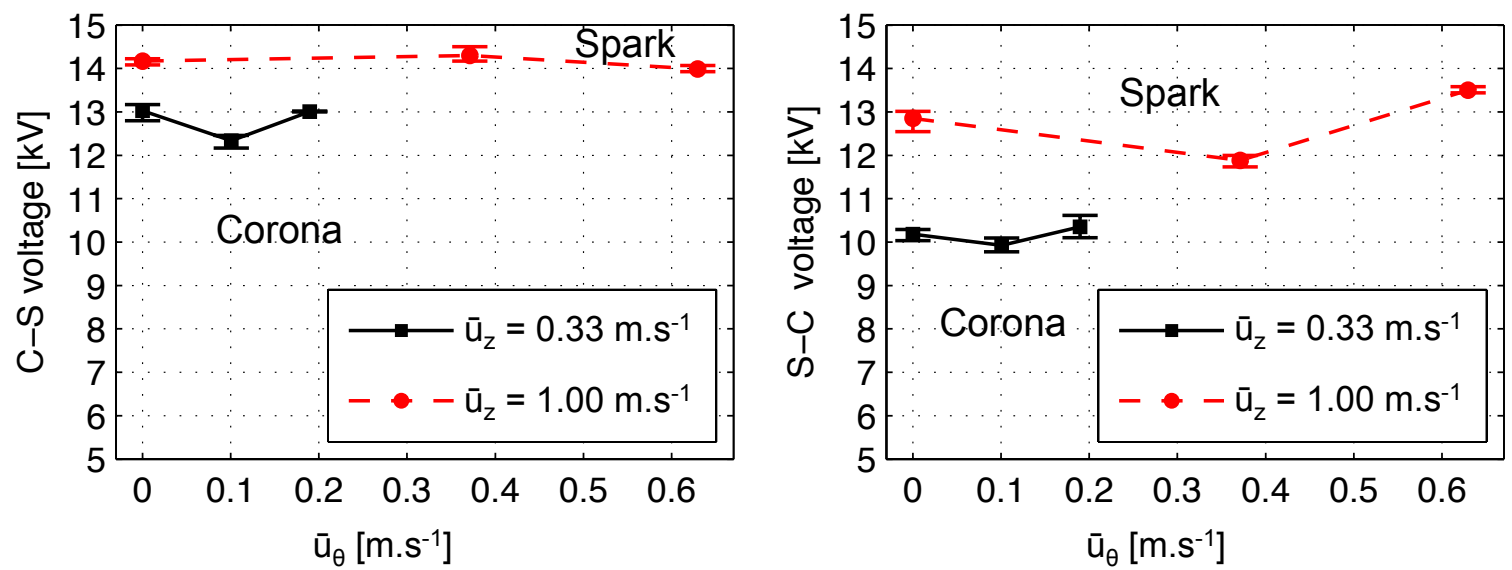

Figure 8. Voltage values for C-S (left) and for S-C (right) transitions. Two bulk flow velocities, $\bar{u}_{z}$, are considered: $0.33 \mathrm{~m} \cdot \mathrm{s}^{-1}$ (solid black) and $1.00 \mathrm{~m} \cdot \mathrm{s}^{-1}$ (dashed red). The PRF of the pulses is constant and set to $30 \mathrm{kHz}$. 
The voltages required to reach the C-S or the $\mathrm{S}-\mathrm{C}$ transition are very similar for the three examined configurations. The difference between the transition voltages for the medium and strong swirling flows is indeed small compared to the absolute values of the voltages. The difference is less than $1.6 \mathrm{kV}$ variation for voltages between 13 and $15 \mathrm{kV}$, corresponding to a relative difference of about $12 \%$. It can be concluded that, in contrast to the NRP spark discharge organization (see Figure 5), there is no significant effect of the azimuthal component of the flow on the C-S and S-C transition voltages. The following experiments will thus be conducted only for non-swirling flows.

\subsection{Harmonically oscillating flow}

Experiments on the effect of low frequency acoustic forcing are conducted here for a total duration of $16 \mathrm{~s}$. The definition of the mean (or bulk) velocity, $\bar{u}_{z}$, is given in Equation 1. The RMS velocity, $u_{z}^{\prime}$, that will be used to estimate the residence time of air particles in the inter-electrode area is expressed as:

$$
u_{z}^{\prime}=\left(\frac{1}{T} \int_{0}^{T}\left(\bar{u}_{z}^{t}-\bar{u}_{z}\right)^{2} d t\right)^{1 / 2}
$$

where $\bar{u}_{z}^{t}$ is the instantaneous reconstructed resulting velocity in the inter-electrode region (see Section 2.3.2) and $T=1 / f_{a c}$ is the forcing period of the harmonic signal.

The NRP regime chart is presented in Figure 9 as a function of the acoustic forcing frequency $f_{a c}$ and the modulation velocity $u_{z}^{\prime}$ when the bulk flow velocity is set to $\bar{u}_{z}=$ $1.42 \mathrm{~m} \cdot \mathrm{s}^{-1}$. In this figure, the $P R F$ is set to $30 \mathrm{kHz}$ and the voltage is fixed to $12.75 \mathrm{kV}$. In the first region, designated by (1) and corresponding to relatively low modulation amplitudes, the NRP plasma discharges are in the corona regime. The second region, designated by (2), corresponds to the NRP spark regime, reached for moderate velocity fluctuations. The third region, designated by (3), is reached when the modulation amplitude is increased further; the NRP discharges return to the corona regime. For forcing frequencies higher than $192 \mathrm{~Hz}$, the NRP spark regime cannot be observed, and the plasma remains in the NRP corona regime, irrespective of the forcing amplitude.

The results presented in the following are given in non-dimensional form: a Strouhal number is defined as:

$$
S t=\frac{f_{a c} \times h}{\bar{u}_{z}}
$$

where $h=1.2 \mathrm{~mm}$ is the height of the inter-electrode area. The RMS velocity of the acoustic forcing, $u_{z}^{\prime}$, is expressed as the relative RMS velocity by dividing it by the mean velocity of the air flow, $\bar{u}_{z}$.

Figure 10 compares the relative RMS velocities, $u_{z}^{\prime} / \bar{u}_{z}$, measured for the C-S (left curves) and S-C (right curves) transitions of NRP discharges for the three experimental conditions defined in Table 2 as a function of the Strouhal number, St. For a bulk injection velocity of $\bar{u}_{z}=1.42 \mathrm{~m} \cdot \mathrm{s}^{-1}$ and a $P R F$ of $30 \mathrm{kHz}$, an air particle is convected, on average, by $47 \mu \mathrm{m}$ between two pulses. By changing the $P R F$ or the mean resulting axial air velocity, the particle displacement between two NRP discharges is altered, 


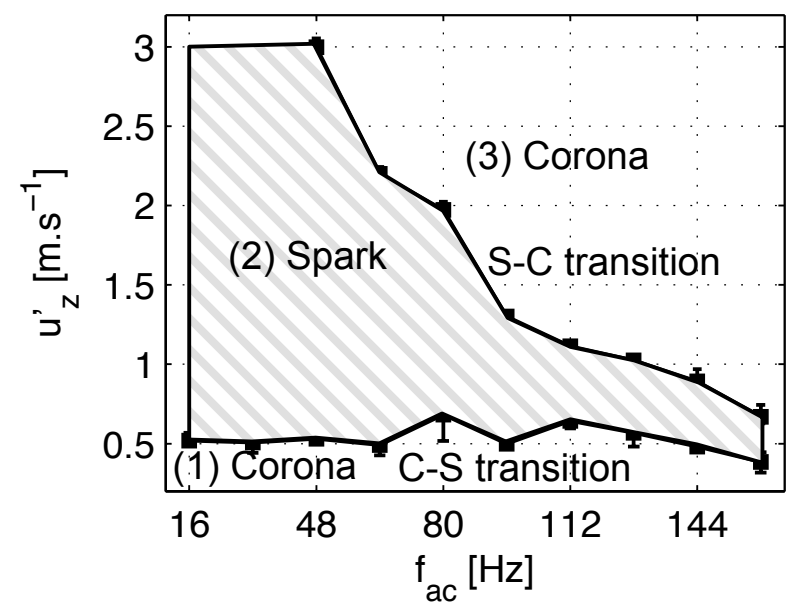

Figure 9. NRP discharges regimes for acoustic forcing frequencies, $f_{a c}$, varying from 16 to $192 \mathrm{~Hz}$ and amplitudes, $u_{z}^{\prime}$, between 0 and $3 \mathrm{~m} \cdot \mathrm{s}^{-1}$. The mean velocity, $\bar{u}_{z}$, is set to $1.42 \mathrm{~m} \cdot \mathrm{s}^{-1}$, the $P R F$ is $30 \mathrm{kHz}$ and the voltage amplitude is $12.75 \mathrm{kV}$.

and the displacement of the previously treated air particles is modified, as explained in Section 3.1. The C-S transition in Figure 10 (left) is reached at a similar relative RMS velocity value over the Strouhal span (corresponding to acoustic frequencies between 16 and $192 \mathrm{~Hz}$ ). The S-C transition curves follow different trend. For low Strouhal numbers (typically, for acoustic frequencies lower than $100 \mathrm{~Hz}$ ) the modulation level required for the $\mathrm{S}-\mathrm{C}$ transition is much larger than at high Strouhal number. The relative RMS velocities for the transitions are similar for each set of experimental conditions.
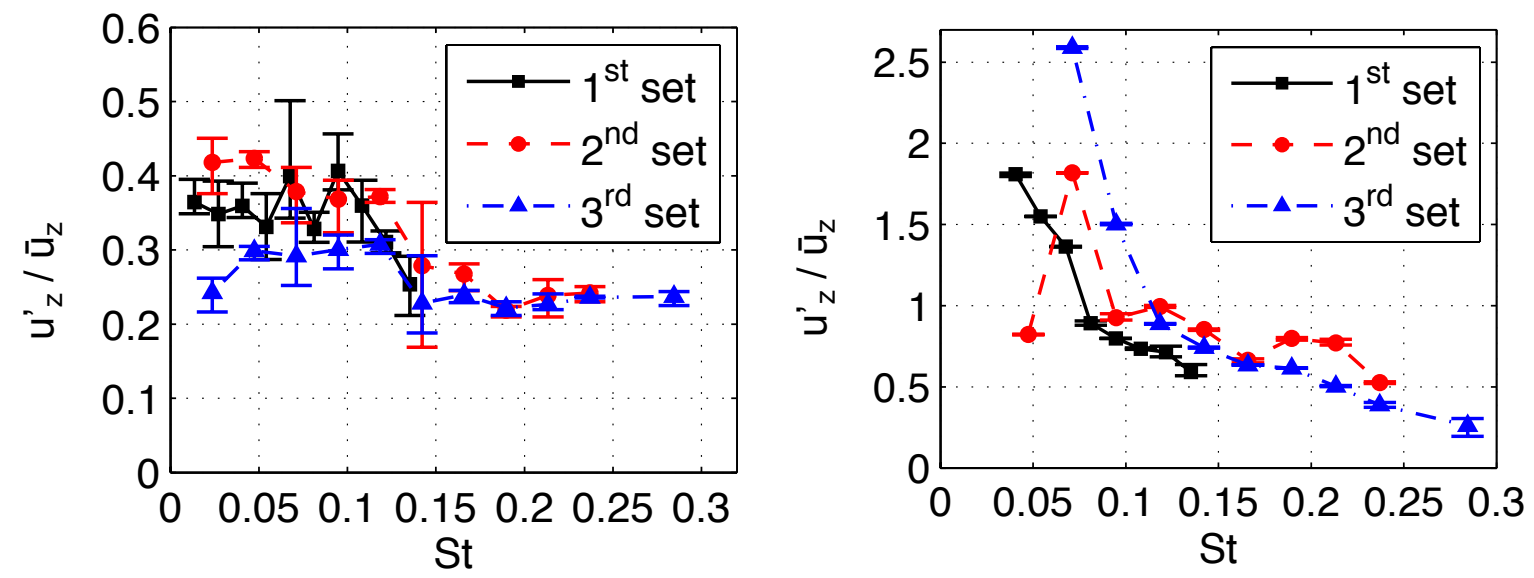

Figure 10. C-S transition (left) and S-C transition (right) curves in terms of the relative RMS velocity and the Strouhal number. The sets of experimental conditions are detailed in Table 2. 


\section{Analysis and discussion}

\subsection{Steady air flow}

It was shown in the previous section that the $\mathrm{C}-\mathrm{S}$ and $\mathrm{S}-\mathrm{C}$ regime transition voltages increase with the bulk flow velocity (Figure 6), supposedly due to a faster transport of the air particles out of the inter-electrode region. Figure 11 shows a schematic of this region that spans over the height $h=1.2 \mathrm{~mm}$, corresponding to the height of the cathode. The mean residence time, $t_{R}$, of an air particle in this region is then given by:

$$
t_{R}=\frac{h}{\bar{u}_{z}} .
$$

The residence time multiplied by the PRF yields the number of NRP pulses to which an air particle is submitted when travelling through the inter-electrode region:

$$
n=t_{R} \times P R F .
$$

Table 3 makes a synthesis, for a PRF of $30 \mathrm{kHz}$, of the residence times $\left(t_{R}\right)$ and number of applied pulses on air particles in the inter-electrode area $(n)$, when in the velocity $\left(\bar{u}_{z}\right)$ range from Figure 6 .

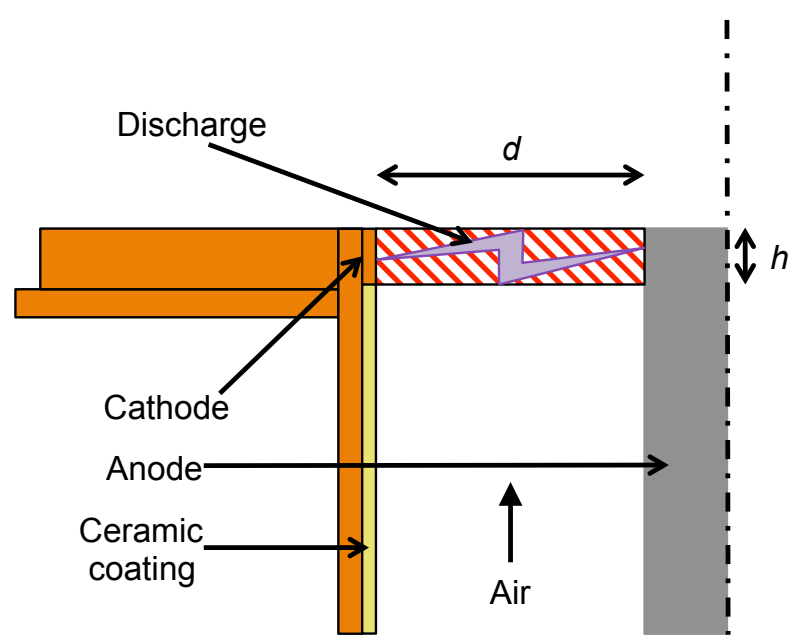

Figure 11. Cross-section of the inter-electrode area at the outlet of the injection tube, where the NRP plasma discharges are generated. The gap distance between the anode and the cathode is $d=7.7 \mathrm{~mm}$ and the height of the inter-electrode area is $h=1.2 \mathrm{~mm}$.

Increasing the convective transport velocity exposes an air particle to less NRP plasma discharges, reducing the cumulative heating and active species production. Increasing the voltage amplitude compensates this effect and increases the heating and active species production induced by individual pulses to reach the transition (Figure 6). It is reasonable to assume that the pulse power required to reach a NRP regime transition is inversely proportional to the mean number of applied pulses on air particles during their transit in the inter-electrode area. The validity of this analysis is verified by turning the parameters in Figure 6 non-dimensional and by comparing them with the data from Figure 7 . The resulting axial air velocity, $\bar{u}_{z}$, is now expressed as the mean 
Table 3. Mean residence times and number of applied pulses on the particles in the inter-electrode area for the studied bulk velocities. Calculations made with Equation (8), with an inter-electrode area height of $1.2 \mathrm{~mm}$ and a $P R F$ of $30 \mathrm{kHz}$.

\begin{tabular}{llr}
\hline $\bar{u}_{z}\left[\mathrm{~m} . \mathrm{s}^{-1}\right]$ & $t_{R}[\mathrm{~ms}]$ & \multicolumn{1}{l}{$n$} \\
\hline 0.33 & 3.64 & 109 \\
\hline 0.66 & 1.82 & 55 \\
\hline 1.00 & 1.20 & 36 \\
\hline 1.33 & 0.90 & 27 \\
\hline
\end{tabular}

number of high-voltage pulses applied on an air particle in the inter-electrode area, $n$ (see Equation 9). The inter-electrode voltage for C-S transition, $U$, leads to a nondimensional power of the pulses, $P^{*}$, defined as:

$$
P^{*}=\frac{U^{2} / R_{\text {air }}}{U_{0}^{2} / R_{\text {air }}}
$$

where $R_{\text {air }}=1.19 \times 10^{16} \Omega$ is the electrical resistance of the considered volume of air (see Figure 1) under atmospheric conditions, and $U_{0}$ is the inter-electrode voltage required to reach C-S transition in quiescent air. Plotting the product of the non-dimensional pulse power and the number of pulses, $P^{*} \times n$, as a function of the number of pulses, $n$ (see Figure 12) illustrates the linear evolution between the two quantities. The data at $\bar{u}_{z}=0 \mathrm{~m} \cdot \mathrm{s}^{-1}$ have not been included because the number of applied pulses in a quiescent air is hard to quantify (the only air motion results from the thermal expansion of the air following a high-voltage pulse). The data from the two experiments presented in Section 3.1.1 (from Figures 6 and 7) superimpose well.

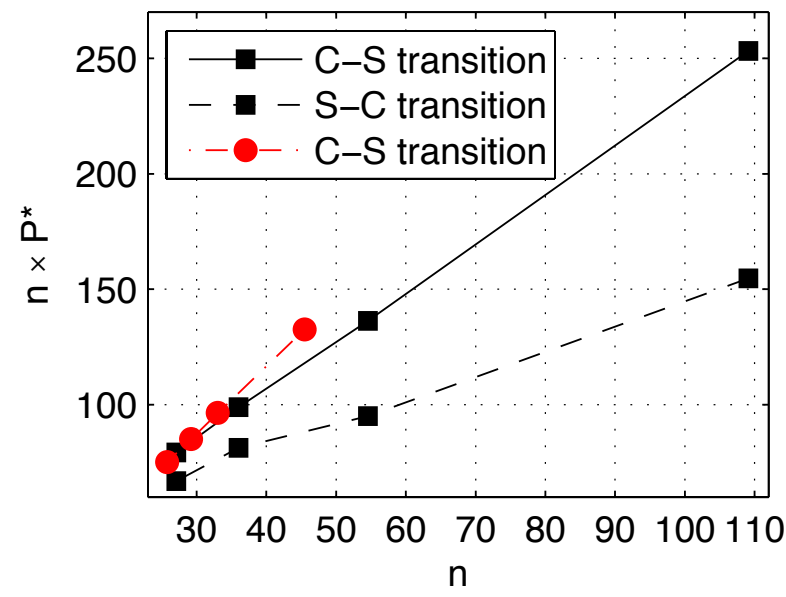

Figure 12. Non-dimensionalized power of the high voltage pulses, $P^{*}$, multiplied by the mean number of applied pulses on an air particle in the inter-electrode area, $n$, as a function of $n$. 
The displayed linear trend represents the following relation between the pulse power, $P^{*}$, and the number of applied pulses on an air particle, $n$ :

$$
P^{*}=A+\frac{B}{n}
$$

where $A=2.52$ and $B=10.95$. Equation 11 implies that an increase of the number of applied pulses on the air particles results in a decrease of the required power to reach C-S transition in the inter-electrode area. This analysis is consistent with the expectation and with the experimental observations, when analyzing the evolution of the C-S transition voltage or of the $P R F$ (see Figures 6 and 7 ) as a function of the axial air velocity.

However, even with a constant voltage, the properties of the air in the interelectrode area change after each high-voltage pulse. The energy deposition is then different for each pulse. Thus, the total energy deposition on an air particle that is required to reach a NRP regime transition, $E_{\text {transition, }}$, is expressed as:

$$
E_{\text {transition }}=\sum_{i=1}^{n} E_{p, i}=\sum_{i=1}^{n} \int_{\frac{i-1}{P R F}}^{\frac{i}{P R F}} U(t) I_{i}(t) d t .
$$

The analysis based on the calculation of the non-dimensional pulse power, $P^{*}$, as a function of the square inter-electrode voltage, $U^{2}$, is rough and the equivalent electrical circuit of the inter-electrode area is not trivial to model. The modeling of $I(t)$ that is necessary to determine the relative impact of $n$ and $U$ on the C-S and S-C transitions will require further investigations, and is not considered in the present work.

Finally, modifying the azimuthal velocity by imparting swirl to the flow does not alter the residence time inside the inter-electrode region so that air particles are still exposed to the same number of pulses, which is consistent with the results in Figure 8.

\subsection{Pressure oscillations induced by acoustic forcing}

The effect of acoustic oscillations on the regime transitions is now discussed. Sound waves are accompanied by small fluctuations in pressure and velocity. Their effects on the formation of the discharges are examined separately.

A variation in pressure induced by the acoustic field alters the reduced electric field $E / N$ that might, in turn, modify the discharge regime transition (see for example [35]):

$$
\frac{E}{N}=\frac{U}{d N} \text {. }
$$

Here, $U$ is the inter-electrode voltage, $d$ the gap distance and $N$ the particle density. Assuming plane wave propagation, sound pressure waves associated with fluctuations of the acoustic velocity with a RMS fluctuation $u_{z}^{\prime}$ in the inter-electrode region correspond to pressure fluctuations $p^{\prime} \simeq Z_{0} u_{z}^{\prime}$, where $Z_{0} \simeq 400 \mathrm{~kg} \cdot \mathrm{m}^{-2} \cdot \mathrm{s}^{-1}$ is the characteristic impedance of air at atmospheric pressure and room temperature. Due to the relatively large values taken by $u_{z}^{\prime} \leq 3 \mathrm{~m} \cdot \mathrm{s}^{-1}$, the corresponding RMS sound pressure levels also reach high values: $p^{\prime} \leq 1200 \mathrm{~Pa}$ (i.e. $155 \mathrm{~dB}$ ). This level of fluctuations is however weak compared to the atmospheric pressure $p^{\prime} / \bar{p} \simeq 10^{-2}$ but could be sufficient to 
explain a NRP regime transition. We therefore performed pressure measurements with a microphone, to check the pressure variation in our experimental setup during acoustic forcing.

Most favorable conditions for spark formation will take place at the instance of lowest density, thus lowest pressure. The minimum peak of the pressure fluctuation was evaluated for a range of acoustic frequencies from 32 to $160 \mathrm{~Hz}$ and for the amplitudes corresponding to C-S and S-C transitions with a bulk velocity of $1.42 \mathrm{~m} . \mathrm{s}^{-1}$ and a PRF of $30 \mathrm{kHz}$. Figure 13 presents the results. The error bars take into account the calibration and the sensitivity of the microphone conditioning amplifier; these were estimated at $5 \%$ of the measured pressure value.

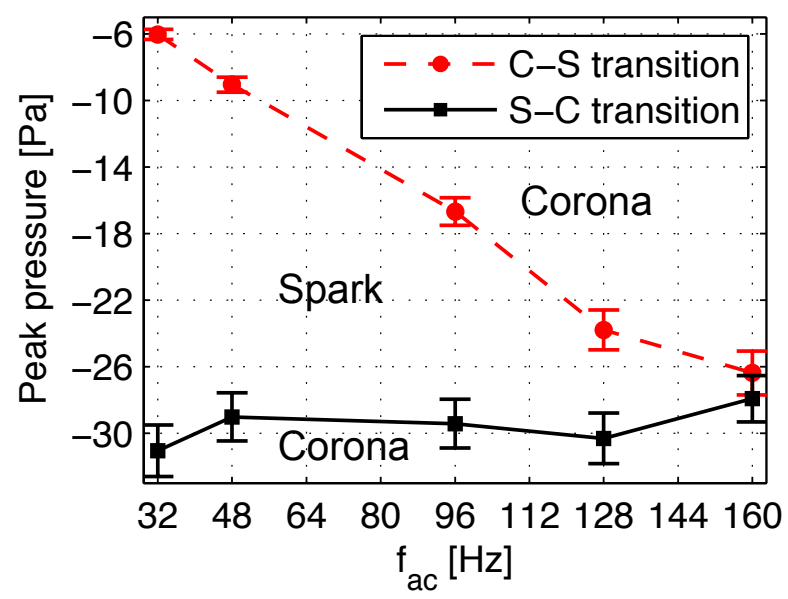

Figure 13. Negative peak pressure measured for C-S transition (dashed red curve) and S-C transition (solid black curve). The harmonic oscillation amplitudes match the ones corresponding to C-S and S-C transitions with a mean velocity of the air flow of $1.42 \mathrm{~m} \cdot \mathrm{s}^{-1}$; pulses at a $P R F$ of $30 \mathrm{kHz}$ and a voltage amplitude of $12.75 \mathrm{kV}$.

The minimum pressure, which should favor NRP spark discharges, actually corresponds to the $\mathrm{S}-\mathrm{C}$ transition. The evolution of the measured pressure is therefore not consistent with the expected trend. Furthermore, the minimum pressure resulting from acoustic forcing lies between 6 and $31 \mathrm{~Pa}$ below the mean pressure (corresponding to a fluctuation between 55 and $62 \mathrm{~dB}$ ), far smaller than the expectations. These measured values are very small compared to the atmospheric pressure $\left(p^{\prime} / \bar{p} \simeq 10^{-4}\right)$ so that the effect of a temporarily decreased pressure on NRP spark discharges formation can be considered negligible. Pressure can therefore be ruled out as a significant factor in the NRP regime transitions observed during acoustic forcing of the flow.

\subsection{Residence times during acoustic forcing}

Experiments presented in Section 3.2 were carried out for different repetition frequencies and air velocities, at a fixed voltage amplitude. For each set of these experimental conditions, the inter-electrode voltage is adjusted so as to be close to the C-S transition. 
Effects of the voltage amplitude are not considered in the following analysis. Since the breakdown phenomenon is highly non-linear with the inter-electrode voltage, the impact is difficult to model, and this problem will be considered in future work. The objective here is to examine whether modifications of the residence time may be used to interpret the transitions observed in Figure 9.

The residence time, $t_{R}$, is defined as the time that a particle located at a boundary of the inter-electrode domain $(z=0$ or $z=h$ ) needs to move out of this area, i.e. go beyond the upper limit $z>h$. The residence time, if the air particle was initially located at $z=0$, is thus implicitly defined by:

$$
h=\int_{t_{1}}^{t_{1}+t_{R}} \bar{u}_{z}^{t} d t,
$$

where $t_{1}$ is an arbitrary time in the acoustic forcing period. If the air particle was initially located at $z=h$, the residence time is:

$$
0=\int_{t_{1}}^{t_{1}+t_{R}} \bar{u}_{z}^{t} d t
$$

supposing that the particles are not convected upstream of the inter-electrode area.

The instantaneous axial velocity of the flow, $\bar{u}_{z}(t)$, can be expressed, in the case of a sine acoustic forcing, as:

$$
\bar{u}_{z}^{t}=\bar{u}_{z}+u_{z}^{\prime} \sqrt{2} \sin \left(\frac{2 \pi}{T} t\right)
$$

where $T=1 / f_{a c}$ is the acoustic period. Because the axial extent of the inter-electrode area is small compared to the acoustic wavelength $\left(C_{a} / f_{a c}\right.$, with $C_{a}$ the speed of sound in air) comprised between 1.77 and $21.25 \mathrm{~m}$ at the low frequencies considered here, the unsteady velocity component can be taken as spatially constant.

Equations (14) and (15) can then be integrated to yield implicit expressions for the residence time:

$$
\begin{aligned}
& t_{R}=\frac{h}{\bar{u}_{z}}+\frac{u_{z}^{\prime}}{\bar{u}_{z}} \frac{T}{\pi \sqrt{2}}\left[\cos \left(\frac{2 \pi}{T}\left(t_{1}+t_{R}\right)\right)-\cos \left(\frac{2 \pi}{T} t_{1}\right)\right], \\
& t_{R}=\frac{u_{z}^{\prime}}{\bar{u}_{z}} \frac{T}{\pi \sqrt{2}}\left[\cos \left(\frac{2 \pi}{T}\left(t_{1}+t_{R}\right)\right)-\cos \left(\frac{2 \pi}{T} t_{1}\right)\right] .
\end{aligned}
$$

Equations (17) and (18) apply for the case that the air particle was initially located respectively at $z=0$ and $z=h$ and show that, for high frequencies of the acoustic forcing (low $T$ ), the effect of the flow modulation will be small so that the residence time is similar to the one without acoustic forcing. The effect of flow modulation on the C-S and S-C transitions therefore decreases with frequency. This is in agreement with the experimental results: it was not possible to obtain NRP spark discharges for frequencies larger than $200 \mathrm{~Hz}$.

In order to find the maximum residence time, $t_{R, \max }$, which occurs for a certain initial acoustic phase, $t_{1}$ can be determined following different models depending on the amplitude of the acoustic oscillations. 


\subsubsection{Case without backflow}

We first consider the case where the oscillation amplitude is smaller or equal to the mean flow velocity $\left(u_{z}^{\prime} \sqrt{2} \leq \bar{u}_{z}\right)$. The model detailed here will be named Model 0 . For this case, an air particle always moves in the direction of the mean flow, and the residence time will be maximum for the following condition (corresponding to a minimum air velocity, see Figure 14):

$$
t_{1}+\frac{t_{R}}{2}=\frac{3}{4} T
$$

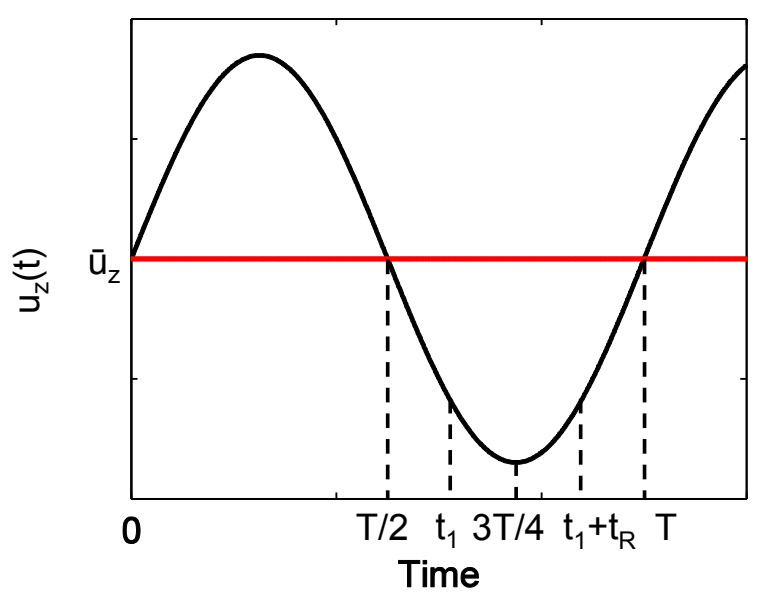

Figure 14. Illustration of the required condition (19) to ensure a maximum residence time of the air particles in the inter-electrode area when $u_{z}^{\prime} \sqrt{2} \leq \bar{u}_{z}$ (Model 0).

Then, for the case where the air particle is initially located at $z=0$, Equation (17) gives:

$$
t_{R, \max }=\frac{h}{\bar{u}_{z}}+\frac{u_{z}^{\prime}}{\bar{u}_{z}} \frac{T}{\pi \sqrt{2}} \sin \left(\frac{\pi}{T} t_{R, \max }\right) .
$$

Figure 15 presents the effect of the acoustic oscillation frequency on the number of applied pulses on air particles for relative RMS velocities $u_{z}^{\prime} / \bar{u}_{z}=0.21,0.35$ and 0.49 (the mean axial velocity is here set to $\bar{u}_{z}=1.42 \mathrm{~m} \cdot \mathrm{s}^{-1}$ ). These values were chosen according to the C-S transition in Figure 9. The effect of the oscillation frequency is not significant. The maximum variation is obtained for a relative amplitude of $u_{z}^{\prime} / \bar{u}_{z}=$ 0.49 , where $n$ only decreases from 80 at $S t=0.01$ to 55 at $S t=0.16$. As a comparison, the number of applied pulses without acoustic forcing is 25 . Thus, for any frequency in the range of 16 to $192 \mathrm{~Hz}$, the acoustic forcing significantly increases the residence time of air particles in the inter-electrode area, even at low amplitudes of forcing.

\subsubsection{Case with backflow}

We now consider the case where the acoustic oscillation amplitude is larger than the mean flow velocity $\left(u_{z}^{\prime} \sqrt{2}>\bar{u}_{z}\right)$. For this case, during a certain part of the acoustic 


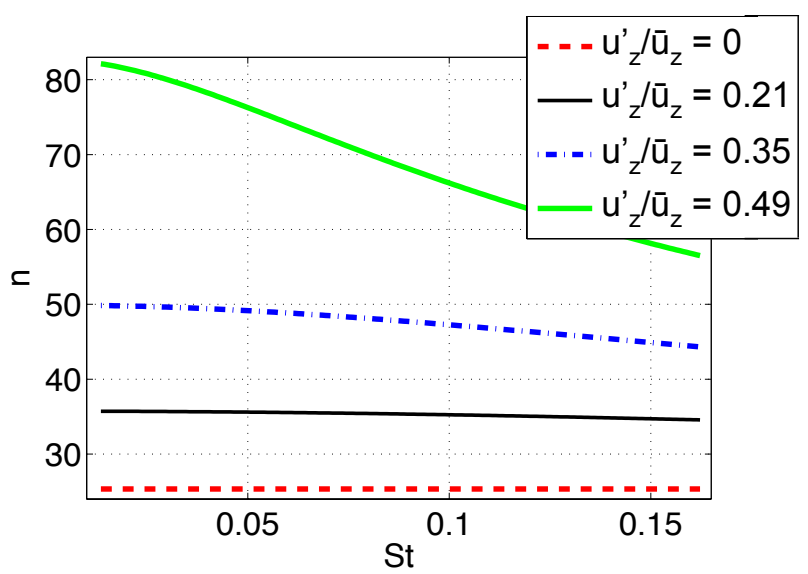

Figure 15. Effect of the frequency of the acoustic forcing on the maximum residence time $t_{R, \max }$, predicted by Equation (20), for low amplitudes of the forcing, corresponding to Model 0 . The mean velocity of the flow, $\bar{u}_{z}$, is $1.42 \mathrm{~m} \cdot \mathrm{s}^{-1}$.

period, the flow velocity is negative and an air particle moves upstream. In order to maximise the residence time, different scenarios are possible, depending on the initial location of the air particle. For an air particle initially located at the lower boundary of the inter-electrode area $(z=0)$, the residence time will be maximum if the particle follows the trajectory depicted in Figure 16 (Model 1). Since the considered period, $T$, of the acoustic forcing is very high compared to the particle residence time in the inter-electrode area, it is not possible for a particle to stay in the inter-electrode area for several acoustic periods. In order to optimize the trajectory (red line), we need to carefully choose $t_{1}$ and $t_{2}$ for each frequency and amplitude of the oscillation. This ideal trajectory will produce the largest residence time, $t_{R, \max }$, that can be obtained by solving the following system of four equations:

$$
\begin{aligned}
& \int_{t_{1}}^{t_{1}+t_{R, \max }} \bar{u}_{z}^{r} d t=h, \\
& \int_{t_{1}}^{t_{2}} \bar{u}_{z}^{r} d t \quad=0, \\
& u_{z}\left(t_{2}\right) \quad=0 \text {, } \\
& \frac{d u_{z}}{d t}\left(t_{2}\right) \quad>0 \text {. }
\end{aligned}
$$

Figure 17 presents the effect of acoustic oscillation amplitude and Strouhal number on the number of applied pulses on a particle initially located at $z=0$. The mean flow velocity is set to $1.42 \mathrm{~m} \cdot \mathrm{s}^{-1}$. The hatched area indicates conditions for which Model 1 cannot be applied, meaning that the red trajectory in Figure 16 cannot be obtained; the trajectory is then similar to the blue one. In order to explain the $\mathrm{S}-\mathrm{C}$ transition, i.e. for high oscillation amplitudes, this model can only be used for elevated frequencies. From 112 to $192 \mathrm{~Hz}$ ( $S t=0.1$ to 0.16 ), the number of applied pulses that can be obtained 


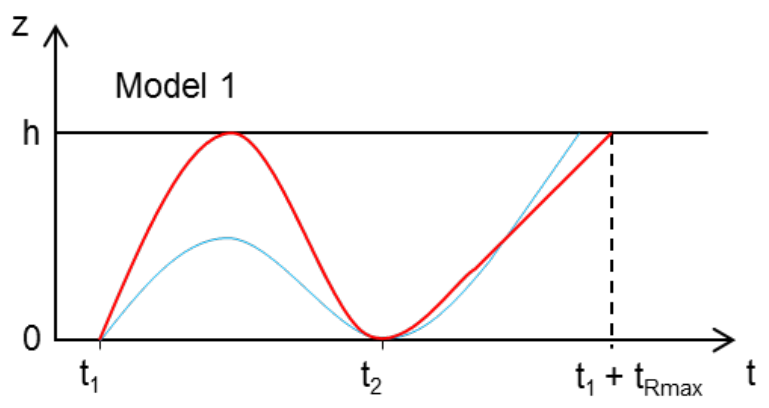

Figure 16. Non-ideal (blue) and ideal (red) air particle trajectories to maximise the residence time of an air particle in the inter-electrode area when it is initially located at $z=0$, for acoustic oscillation amplitudes larger than the mean velocity.

following Model 1 varies from 150 to 100. The corresponding RMS oscillation amplitudes are $1.6 \mathrm{~m} \cdot \mathrm{s}^{-1}$ and $1.8 \mathrm{~m} \cdot \mathrm{s}^{-1}$, hence a relative RMS amplitude between 1.1 and 1.3 . These values are slightly larger than the experimental results presented in Figure 9; however, the tendency is apparent. For high oscillation frequencies, the amplitude has only little effect on the maximum residence time. By increasing or decreasing the mean velocity of the flow, the limit RMS velocity for which Model 1 is valid is displaced respectively to higher or lower RMS velocity values.

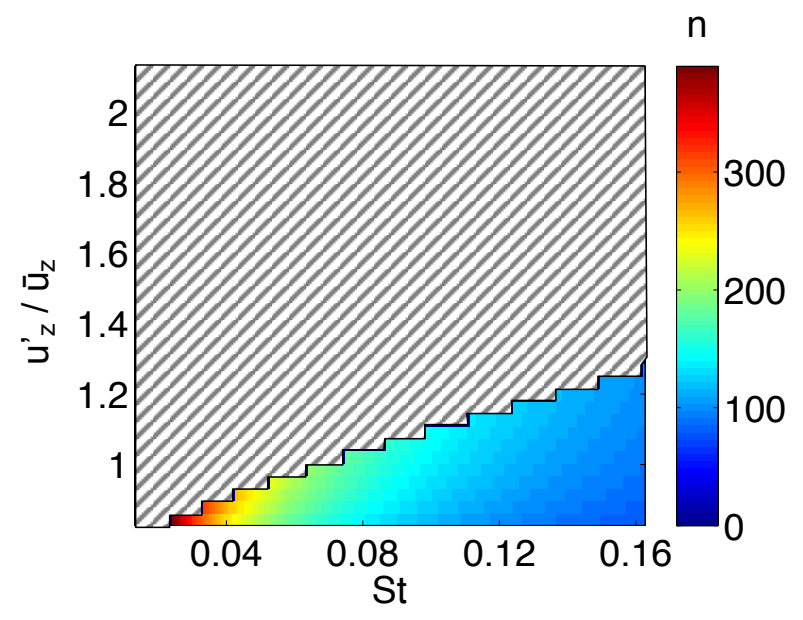

Figure 17. Number of applied pulses on air particles as a function of Strouhal number and relative velocity amplitude following Model 1 from Figure 16. The hatched area corresponds to conditions for which Model 1 is not valid. The mean velocity of the flow is $1.42 \mathrm{~m} \cdot \mathrm{s}^{-1}$.

By now considering an air particle initially located at the upper boundary of the inter-electrode area $(z=h)$, the maximum residence time, $t_{R, \max }$, will be attained if the air particle follows the red trajectory in Figure 18 (Model 2). In this case, the maximum residence time is found by solving the system of equations: 


$$
\begin{aligned}
& \int_{t_{1}}^{t_{1}+t_{R, \max }} \bar{u}_{z}^{r} d t=0, \\
& \int_{t_{1}}^{t_{2}} \bar{u}_{z}^{r} d t \quad=-h, \\
& u_{z}\left(t_{2}\right) \quad=0 \text {, } \\
& \frac{d u_{z}}{d t}\left(t_{2}\right) \quad>0 \text {. }
\end{aligned}
$$

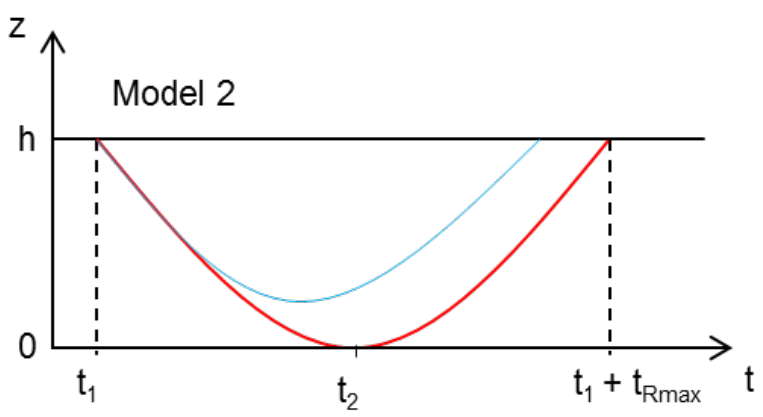

Figure 18. Non-ideal (blue) and ideal (red) air particle trajectories to maximise the residence time of an air particle in the inter-electrode area when it is initially located at $z=h$, for acoustic oscillation amplitudes larger than the mean velocity.

Figure 19 presents the effect of relative oscillation amplitude and Strouhal number on the number of applied pulses on a particle initially located at $z=h$ with a mean air velocity of $1.42 \mathrm{~m} . \mathrm{s}^{-1}$. The hatched area indicates conditions for which Model 2 cannot be applied, i.e. the red trajectory in Figure 18 cannot be obtained (the trajectory then corresponds to the blue curve of the figure). A given number of pulses at low Strouhal number (for example, 120 pulses in cyan) follows the same trend as the $\mathrm{S}-\mathrm{C}$ transition in Figure 9. Changing the mean velocity of the air flow induces the same displacement of the limit RMS velocity for which Model 2 is valid as in Figure 17.

The whole effect of acoustic forcing on the maximum residence time is finally considered by combining the results for low oscillation amplitudes (Model 0) and for the case with backflow (Model 1 and Model 2). Table 4 summarizes the different considered conditions and the maximum residence times that an air particle can present, depending on the amplitude and the frequency of the forcing. Figure 20 shows isolines for a number of 90 pulses (residence time of $3 \mathrm{~ms}$ ), obtained from a combination of the individual models (black curves). This value of 90 pulses provides the best fit with the experimental values (red markers) for the C-S and the $\mathrm{S}-\mathrm{C}$ transitions. The qualitative structure in the frequency-amplitude domain looks very similar to the experimentally obtained transition curves in Figure 9. It can therefore be concluded that the dominant effect of acoustic oscillations on the C-S and S-C transitions is probably linked to a change in the residence time of the air in the inter-electrode area. An interesting topic would be the establishment of the role of the inter-electrode voltage in both the C-S and 


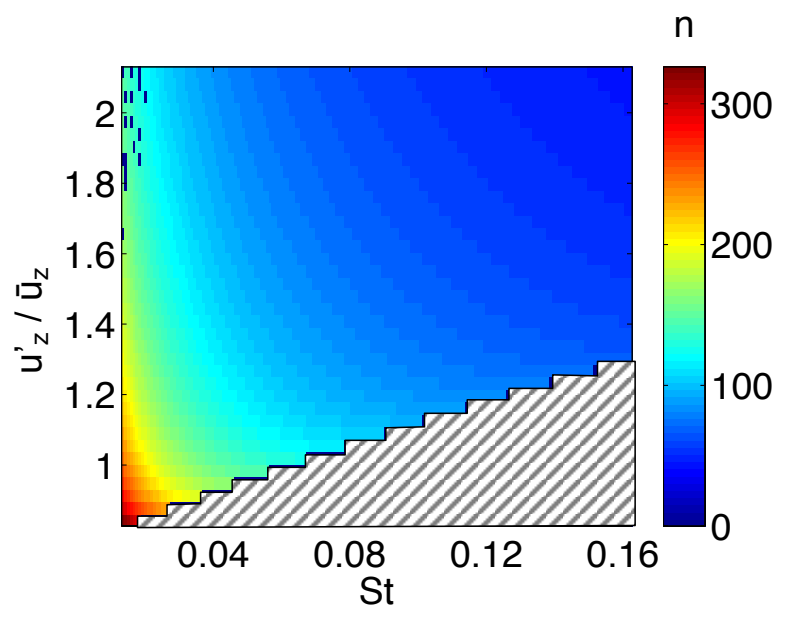

Figure 19. Maximum residence time as a function of oscillation frequency and relative velocity amplitude following Model 2 from Figure 18. The hatched area corresponds to conditions for which Model 2 is not valid. The mean velocity of the flow is $1.42 \mathrm{~m} \cdot \mathrm{s}^{-1}$.

the $\mathrm{S}-\mathrm{C}$ transitions and its link with the particle residence time in the inter-electrode area. This topic could be the focus of further studies.

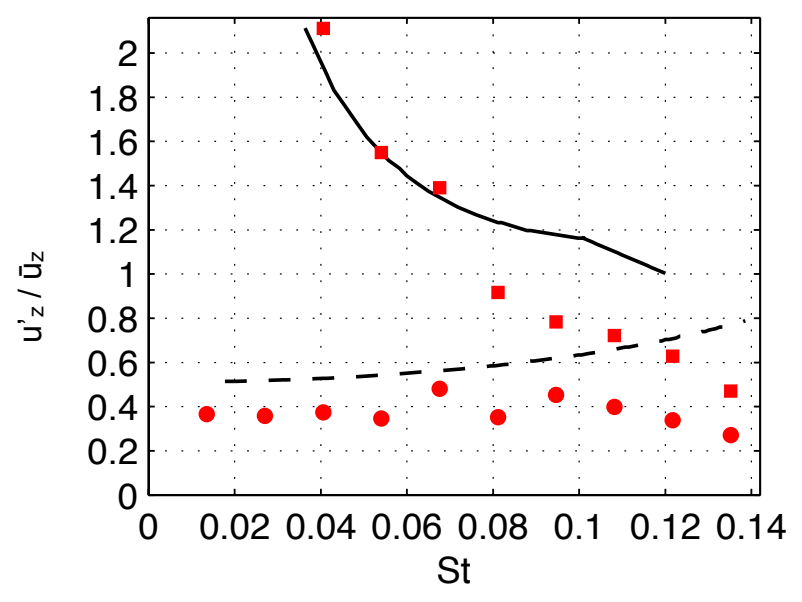

Figure 20. Isolines corresponding to a number of 90 pulses applied to the air particles, obtained by combining modeling results (black curves) for low-amplitude (Model 0, dashed line) and for high-amplitude forcing (Model 1 and Model 2, solid line), for a mean velocity of the flow $\bar{u}_{z}=1.42 \mathrm{~m} \cdot \mathrm{s}^{-1}$, compared to experimental measurements (red markers) for a $P R F$ of $30 \mathrm{kHz}$, voltage pulses amplitude of $12.75 \mathrm{kV}$ and a mean air velocity of $1.42 \mathrm{~m} \cdot \mathrm{s}^{-1}$. Number of pulses larger than 90 are found to the left of the lines. 
Table 4. Summary of the conditions and the equations of Models 0,1 and 2.

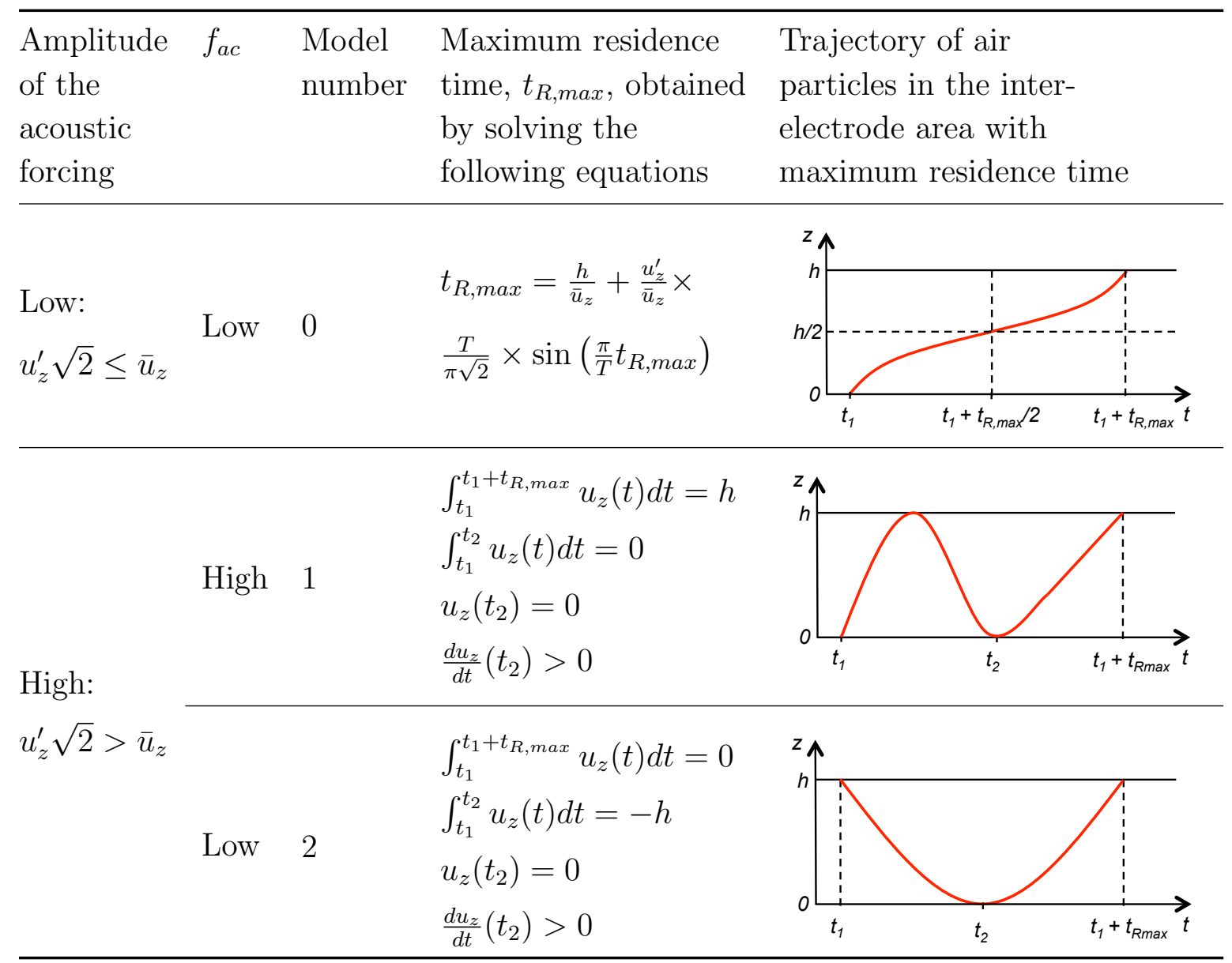

\section{Conclusion}

The effect of steady and harmonically oscillating flow on the regime transitions of NRP discharges was experimentally investigated in a pin-annular configuration. The importance of the axial air velocity on the C-S and S-C regime transition voltages was demonstrated. The azimuthal velocity component was found to have a negligible effect on the regime transitions, for the investigated range of velocities. These experimental observations can be explained by analyzing the cumulative effect of repetitive nanosecond discharges and the residence time of air particles in the interelectrode area.

In addition, the effect of harmonic oscillations superimposed to the mean steady flow was investigated. For a frequency range from 16 to $192 \mathrm{~Hz}$, a moderate oscillation amplitude can promote the NRP spark regime. Higher oscillation amplitudes, on the other hand, promote the NRP corona regime. The oscillation amplitude at which spark-to-corona transition occurs is strongly frequency dependent. The dependence of the regime transitions on the oscillation amplitude and frequency is explained by their effect on the residence time of air particles in the inter-electrode area. A model 
for the maximum residence time under the effect of acoustic oscillations was built and qualitatively captures the experimentally observed regime transitions in the frequencyamplitude space.

A further study would consist of extending the range of velocities, voltage amplitudes and PRF implemented, as well as testing new electrode configurations. The objectives of these studies would be to reproduce the observed phenomena and evaluate the main non-dimensional quantities governing the mechanisms. These results could then be used to create a predictive model, based on the established significant non-dimensional values.

\section{Acknowledgements}

This study has been supported by the Agence Nationale de la Recherche (ANR) and the Deutsche Forschungsgemeinschaft (DFG) through the DRACO project (grant numbers ANR-13-IS09-0004, MO 2551/1). The authors would also like to thank the École Normale Supérieure (ENS) de Cachan, for the stipend of Sylvain Heitz.

\section{References}

[1] Z. Kovalová, M. Zahoran, A. Zahoranová and Z. Machata 2014 Streptococci biofilm decontamination on teeth by low-temperature air plasma of DC corona discharges J. Phys. D: Appl. Phys. 47224014

[2] G. Collet, E. Robert, A. Lenoir, M. Vandamme, T. Darny, S. Dozias, C. Kieda and J. M. Pouvesle 2014 Plasma jet-induced tissue oxygenation: potentialities for new therapeutic strategies Plasma Sources Sci. Technol. 23012005

[3] M. G. Kong, G. Kroesen, G. Morfill, T. Nosenko, T. Shimizu, J. van Dijk and J. L. Zimmermann 2009 Plasma medicine: an introductory review New J. Phys. 11115012

[4] A. M. Hirst, F. M. Frame, N. J. Maitland, D. O'Connell 2014 Low temperature plasma: a novel focal therapy for localized prostate cancer? BioMed Research International 2014878319

[5] F. Massines, C. Sarra-Bournet, F. Franelli, N. Naude and N. Gherardi 2012 Atmospheric pressure low temperature direct plasma technology: status and challenges for thin film deposition Plasma Processes Polym. 9 1041-1073

[6] S. Stauss, Y. Imanishi, H. Miyazoe and K. Terashima 2010 High rate deposition of ZnO thin films by a small-scale inductively coupled argon plasma generated in open air J. Phys. D: Appl. Phys. 43155203

[7] T. Nozaki, A. Hattori and K. Okazaki 2004 Partial oxidation of methane using a microscale nonequilibrium plasma reactor Catal. Today 98 607-616

[8] X. Zhang and M. S. Cha 2015 Partial oxidation of methane in a temperature-controlled dielectric barrier discharge reactor Proc. Combust. Inst 35 3447-3454

[9] N. Mericam-Bourdet, M. J. Kirkpatrick, F. Tuvache, D. Frochot and E. Odic 2012 Effect of voltage waveform on dielectric barrier discharge ozone production efficiency Eur. Phys. J. Appl. Phys. 5730801

[10] A. Starikovskiy and N. Aleksandrov 2013 Plasma-assisted ignition and combustion Prog. Energy Combust. Sci. 39 61-110

[11] I. V. Adamovich, I. Choi, N. Jiang, J. H. Kim, S. Keshav, W. R. Lempert, E. Mintusov, M. Nishihara, M. Samimy and M. Uddi 2009 Plasma assisted ignition and high-speed flow control: non-thermal and thermal effects Plasma Sources Sci. Technol. 18034018 
[12] D. A. Lacoste, J. P. Moeck, D. Durox, C. O. Laux and T. Schuller 2013 Effect of nanosecond repetitively pulsed discharges on the dynamics of a swirl-stabilized lean premixed flame J. Eng. Gas Turbines Power 135101501

[13] D. F. Colas, A. Ferret, D. Z. Pai, D. A. Lacoste and C. O. Laux 2010 Ionic wind generation by a wire-cylinder-plate corona discharge in air at atmospheric pressure J. Appl. Phys. 108103306

[14] M. Rickard, D. Dunn-Rankin, F. Weinberg and F. Carleton 2005 Characterization of ionic wind velocity J. Electrostat. 63 711-716

[15] A. Debien, N. Benard and E. Moreau 2012 Streamer inhibition for improving force and electric wind produced by DBD actuators J. Phys. D: Appl. Phys. 45215201

[16] A. V. Likhanskii, M. N. Schneider, S. O. Macheret and R. B. Miles 2008 Modeling of dielectric barrier discharge plasma actuator in air J. Appl. Phys. 103053305

[17] Y. Lagmich, Th. Callegari, L. C. Pitchford and J. P. Boeuf 2008 Model description of surface dielectric barrier discharges for flow control J. Phys. D: Appl. Phys. 41095205

[18] V. F. Kopiev, Y. S. Akishev, I. V. Belyaev, N. K. Berezhetskaya, V. A. Bityurin, G. A. Faranosov, M. E. Grushin, A. I. Klimov, V. A. Kopiev, I. A. Kossyi, I. A. Moralev, N. N. Ostrikov, M. I. Taktakishvili, N. I. Trushkin and M. Y. Zaytsev 2014 Instability wave control in turbulent jet by plasma actuators J. Phys. D: Appl. Phys. 47505201

[19] D. A. Xu, D. A. Lacoste, D. L. Rusterholtz, P. Q. Elias, G. D. Stancu and C. O. Laux 2011 Experimental study on the hydrodynamic expansion following a nanosecond repetitively pulsed discharge in air Appl. Phys. Lett. 99121502

[20] R. F. Haley and P. R. Smy 1989 Electrically induced turbulence - the short duration spark $J$. Phys. D: Appl. Phys. 22 258-265

[21] N. A. Evans 1967 Effect of flow velocity on a single, controlled glow discharge in cesium-seeded argon AIAA Journal 5 1908-1910

[22] A. Bogaerts, A. Okhrimosckyy and R. Gijbels 2002 Calculation of the gas flow and its effect on the plasma characteristics for a modified Grimm-type glow discharge cell J. Anal. At. Spectrom. 17 1076-1082

[23] S. Z. Li, W. T. Huang and D. Wuang 2009 The effect of gas flow on argon plasma discharge generated with a single-electrode configuration at atmospheric pressure Phys. Plasmas 16093501

[24] P. Chabert, A. Rousseau, G. Gousset and P. Leprince 1998 On the influence of the gas velocity on dissociation degree and gas temperature in a flowing microwave hydrogen discharge $J$. Appl. Phys. 84 161-167

[25] A. Jaworek and A. Krupa 1996 Corona discharge from a multipoint electrode in flowing air $J$. Electrostat. 38 187-197

[26] R. Pereira, D. Ragni and M. Kotsonis 2014 Effect of external flow velocity on momentum transfer of dielectric barrier discharge plasma actuators J. Appl. Phys. 116103301

[27] C. Zhang, T. Shao, P. Yan, Y. Zhou 2014 Nanosecond-pulse gliding discharges between point-topoint electrodes in open air Plasma Sources Sci. Technol. 23035004

[28] R. Bálek, M. Červenka and S. Pekárek 2014 Acoustic field effects on a negative corona discharge Plasma Sources Sci. Technol. 23035005

[29] D. Z. Pai, D. A. Lacoste and C. O. Laux 2010 Transitions between corona, glow and spark regimes of nanosecond repetitively pulsed discharges in air at atmospheric pressure J. Appl. Phys. 107 093303

[30] A.K. Gupta, D.G. Lilley, N. Syred 1984 Swirl Flows Abacus Press, Kent

[31] C. Zhang, T. Shao, Z. Zhou, W. Yang, V.F. Tarasenko, P. Yan 2014 Coaxial diffuse discharges driven by repetitive nanosecond pulses at different air pressures IEEE Trans. Plasma Sci. 42(10) 2378-2379

[32] S.V. Pancheshnyi, D.A. Lacoste, A. Bourdon, C.O. Laux 2006 Ignition of propane-air mixtures by a repetitively pulsed nanosecond discharge IEEE Trans. Plasma Sci. 34(6) 2478-2487

[33] T. Shao, G.S. Sun, P. Yan, J. Wang, W.Q. Yuan, S.C. Zhang 2007 Experimental study of polarity dependence in repetitive nanosecond-pulse breakdown Chinese Phys. 16(3) 778-783 
[34] D. Z. Pai, D. A. Lacoste and C. O. Laux 2010 Nanosecond repetitively pulsed discharges in air at atmospheric pressure - the spark regime Plasma Sources Sci. Technol. 19065015

[35] D. L. Rusterholtz, D. A. Lacoste, G. D. Stancu, D. Z. Pai and C. O. Laux 2013 Ultrafast heating and oxygen dissociation in atmospheric pressure air by nanosecond repetitively pulsed discharges J. Phys. D: Appl. Phys. 46464010

[36] C. Zhang, T. Shao, H. Ma, D. Zhang, C. Ren, P. Yan, V. Tarasenko, E. Schamiloglu 2013 Experimental study on conduction current of positive nanosecond-pulse diffuse discharge at atmospheric pressure IEEE Trans. Dielectrics Electr. Insul. 20(4) 1304-1314 